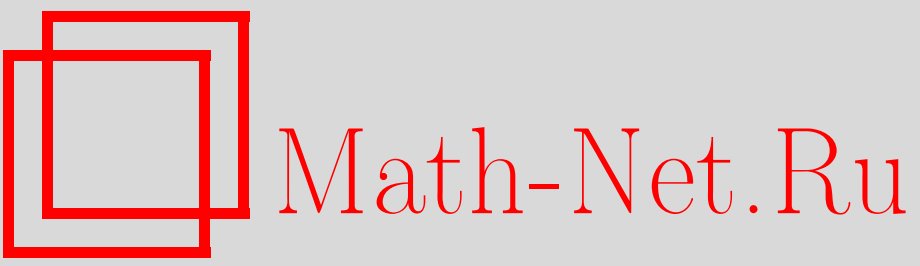

Ан. А. Новиков, П. А. Новиков, Локально наиболее мощные последовательные критерии проверки простых гипотез против односторонних альтернатив для независимых наблюдений, Теория вероятн. и ее примен., 2011, том 56, выпуск $3,449-477$

DOI: https://doi.org/10.4213/tvp4402

Использование Общероссийского математического портала Math-Net.Ru подразумевает, что вы прочитали и согласны с пользовательским соглашением

http://www.mathnet.ru/rus/agreement

Параметры загрузки:

IP: 3.85 .5 .30

26 апреля 2023 г., 17:40:24 
ТЕОРИЯ ВЕРОЯТНОСТЕЙ

Том 56

И ЕЕ ПРИМЕНЕНИЯ

Выпуск 3

2011

(c) 2011 г. НОВИКОВ АН. А. ${ }^{*}$ НОВИКОВ П. А.**

\section{ЛОКАЛЬНО НАИБОЛЕЕ МОЩНЫЕ ПОСЛЕДОВАТЕЛЬНЫЕ КРИТЕРИИ ПРОВЕРКИ ПРОСТЫХ ГИПОТЕЗ ПРОТИВ ОДНОСТОРОННИХ АЛЬТЕРНАТИВ ДЛЯ НЕЗАВИСИМЫХ НАБЛЮДЕНИЙ}

Пусть наблюдается случайный процесс с независимыми значениями $X_{1}, \ldots, X_{n}, \ldots$, распределение которого, $\mathbf{P}_{\theta}$, зависит от неизвестного параметра $\theta$. В данной статье рассматривается задача последовательной проверки простой гипотезы $H_{0}: \theta=\theta_{0}$ против сложной альтернативы $H_{1}: \theta>\theta_{0}$, где $\theta_{0}$ - некоторое фиксированное значение параметра.

В первой части данной работы мы приводим условия дифференцируемости (в $\left.\theta_{0}\right)$ функции мощности любого последовательного критерия, а также получаем неравенства информационного типа, связывающие средний объем выборки с вероятностью ошибки первого рода и производной функции мощности последовательных критериев.

Во второй части работы мы даем характеризацию структуры локально наиболее мощных в смысле Берка (Ann. Statist., 1975, v. 3, p. 373) последовательных критериев в данной задаче (максимизирующих производную функции мощности при заданных ограничениях на вероятность ошибки первого рода и средний объем выборки).

Ключевые слова и фразы: последовательный анализ, проверка гипотез, односторонняя альтернатива, последовательный критерий, локально наиболее мощный критерий, оптимальные последовательные решения.

1. Введение. Пусть $X_{1}, \ldots, X_{n}, \ldots$ - случайный процесс с независимыми значениями, распределение которого, $\mathbf{P}_{\theta}$, зависит от неизвестного параметра $\theta, \theta \in \Theta$, где $\Theta$ - открытое подмножество числовой прямой. Рассматривается задача проверки простой гипотезы $H_{0}: \theta=\theta_{0}$ против сложной альтернативы $H_{1}: \theta>\theta_{0}$, где $\theta_{0} \in \Theta$ - некоторое фиксированное значение параметра. Основная цель данной работы - ха-

*Departamento de Matemáticas, Universidad Autónoma Metropolitana-Iztapalapa, San Rafael Atlixco 186, col. Vicentina, C.P. 09340, México D.F., México; e-mail: an@xanum.uam.mx

** Казанский (Приволжский) федеральный университет, НОЦ «Анализ», ул. Кремлевская, 18, Казань, Россия, 420008; e-mail: pnovi@mail.ru 
рактеризация структуры локально наиболее мощных в смысле Берка [8] последовательных критериев в данной задаче.

В определениях и обозначениях, связанных с последовательными критериями проверки гипотез, будем придерживаться работы [21] (см. также [25], [10], [2], [23], [9], [20] среди прочих).

В частности, будем говорить, что пара $(\psi, \phi)$ - последовательный критерий проверки гипотез с (рандомизированным) правилом остановки $\psi$ и (рандомизированным) решающим правилом $\phi$, если

$$
\psi=\left(\psi_{1}, \ldots, \psi_{n}, \ldots\right) \quad \text { и } \quad \phi=\left(\phi_{1}, \ldots, \phi_{n}, \ldots\right),
$$

где функции

$$
\psi_{n}=\psi_{n}\left(x_{1}, \ldots, x_{n}\right) \quad \text { и } \quad \phi_{n}=\phi_{n}\left(x_{1}, \ldots, x_{n}\right)
$$

измеримы и принимают значения в $[0,1]$ для всех $n=1,2, \ldots$.

На каждом этапе $n=1,2, \ldots$ значение $\psi_{n}\left(x_{1}, \ldots, x_{n}\right)$ понимается как условная вероятность остановиться (и перейти к принятию решения) при условии, что эксперимент дошел до этапа $n$ и что наблюдения процесса, полученные до этого этапа включительно, суть $\left(x_{1}, \ldots, x_{n}\right)$. Правила $\psi_{1}, \psi_{2}, \ldots$ применяются последовательно, пока эксперимент на некотором этапе не остановится.

Предполагается, что при остановке на этапе $n \geqslant 1$ для принятия решения используется решающее правило $\phi_{n}$. Значение $\phi_{n}\left(x_{1}, \ldots, x_{n}\right)$ понимается как условная вероятность отклонить нулевую гипотезу $H_{0}$ при наблюдениях $\left(x_{1}, \ldots, x_{n}\right)$.

В соответствии с вышеописанной процедурой правило остановки $\psi$ порождает случайную величину $\tau_{\psi}$ (момент остановки), имеющую распределение

$$
\mathbf{P}_{\theta}\left(\tau_{\psi}=n\right)=\mathbf{E}_{\theta}\left(1-\psi_{1}\right)\left(1-\psi_{2}\right) \cdots\left(1-\psi_{n-1}\right) \psi_{n}, \quad n=1,2, \ldots
$$

Здесь и далее $\mathbf{E}_{\theta}$ означает математическое ожидание относительно распределения $\mathbf{P}_{\theta}$ процесса $X_{1}, X_{2}, \ldots$.

В (1) предполагается, что $\psi_{n}=\psi_{n}\left(X_{1}, \ldots, X_{n}\right)$, хотя первоначально мы определили $\psi_{n}$ как $\psi_{n}\left(x_{1}, \ldots, x_{n}\right)$. Мы используем эту «двойственность» в интерпретации любой функции от наблюдений на протяжении всей статьи, применяя следующее правило, которое делает ее интерпретацию однозначной. Если $F_{n}$ - любая функция от наблюдений $\left(F_{n}=F_{n}\left(x_{1}, \ldots, x_{n}\right)\right.$ или $\left.F_{n}=F_{n}\left(X_{1}, \ldots, X_{n}\right)\right)$ и ее аргументы опущены, то:

- если $F_{n}$ стоит под знаком вероятности или математического ожидания, то это $-F_{n}\left(X_{1}, \ldots, X_{n}\right)$,

- в противном случае $F_{n}$ обозначает $F_{n}\left(x_{1}, \ldots, x_{n}\right)$. 
Для характеризации продолжительности последовательного эксперимента используется величина среднего объема выборки:

$$
\mathscr{N}_{\theta}(\psi)=\mathbf{E}_{\theta} \tau_{\psi}= \begin{cases}\sum_{n=1}^{\infty} n \mathbf{P}_{\theta}\left(\tau_{\psi}=n\right), & \text { если } \mathbf{P}_{\theta}\left(\tau_{\psi}<\infty\right)=1, \\ \infty & \text { в противном случае }\end{cases}
$$

Для последовательного критерия $(\psi, \phi)$ определим функцию мощности в $\theta$ формулой

$$
\beta_{\theta}(\psi, \phi)=\mathbf{P}_{\theta}\left(\text { отклонить } H_{0}\right)=\sum_{n=1}^{\infty} \mathbf{E}_{\theta}\left(1-\psi_{1}\right) \cdots\left(1-\psi_{n-1}\right) \psi_{n} \phi_{n} .
$$

Вероятность ошибки I рода критерия $(\psi, \phi)$ определяется как

$$
\alpha(\psi, \phi)=\beta_{\theta_{0}}(\psi, \phi) .
$$

Основная цель данной работы - характеризация критериев, максимизирующих производную функции мощности в $\theta=\theta_{0}, \dot{\beta}_{\theta_{0}}(\psi, \phi)$, в классе всех последовательных критериев $(\psi, \phi)$ таких, что

$$
\alpha(\psi, \phi) \leqslant \alpha \quad \text { и } \quad \mathscr{N}_{\theta_{0}}(\psi) \leqslant \mathscr{N},
$$

где $\alpha \in[0,1)$ и $\mathscr{N} \geqslant 1-$ некоторые ограничения. Если такой критерий существует, то он называется локально наиболее мощным критерием (см. [8], [22]).

Мы используем довольно общий метод, разработанный первоначально для проверки двух простых гипотез (см. [20]) и распространённый затем на задачу проверки многих гипотез (см. [19]), на общие проблемы последовательных байесовских статистических решений (см. [17], [18]) и на задачи построения локально наиболее мощных критериев (см. [21], [4]).

2. Предположения и обозначения. Предположим, что $X_{i}$ имеет «функцию плотности» $f_{\theta, i}$ (производная Радона-Никодима ее распределения) относительно некоторой сигма-конечной меры $\mu$ на пространстве «значений» $X_{i}, i=1,2, \ldots$.

Вследствие независимости наблюдений для любого $n=1,2, \ldots$ «вектор» $\left(X_{1}, \ldots X_{n}\right)$ первых $n$ наблюдений имеет «плотность»

$$
f_{\theta}^{n}\left(x_{1}, \ldots, x_{n}\right)=\prod_{i=1}^{n} f_{\theta, i}\left(x_{i}\right)
$$

относительно произведения мер

$$
\mu^{n}=\mu \otimes \cdots \otimes \mu \quad(n \text { раз }) .
$$


Будем предполагать (когда это потребуется), что выполнены следующие условия.

Пусть

$$
I_{j}\left(\theta_{0}, \theta_{1}\right)=\mathbf{E}_{\theta_{0}} \ln \frac{f_{\theta_{0}, j}\left(X_{j}\right)}{f_{\theta_{1}, j}\left(X_{j}\right)}
$$

- различающая информация по Кульбаку-Лейблеру между распределенями $X_{j}$ при $\theta=\theta_{0}$ и $\theta=\theta_{1}, j=1,2, \ldots$.

У с л о в и е 1 . Существуют такие $\delta>0$ и $0<\gamma_{1}<\infty$, что

$$
I_{j}\left(\theta_{0}, \theta\right)\left(\theta-\theta_{0}\right)^{-2} \leqslant \gamma_{1}
$$

для всех $j=1,2, \ldots$ и для всех $\left|\theta-\theta_{0}\right| \leqslant \delta$.

Для независимых одинаково распределенных наблюдений условие 1 совпадает с предположением 1 в [8].

У с л о в и е 2 . Для любого $j \geqslant 1$ существует такая интегрируемая (относительно меры $\mu$ ) функция $\dot{f}_{\theta_{0}, j}$, что

$$
\int\left|f_{\theta, j}-f_{\theta_{0}, j}-\left(\theta-\theta_{0}\right) \dot{f}_{\theta_{0}, j}\right| d \mu=o\left(\theta-\theta_{0}\right)
$$

при $\theta \rightarrow \theta_{0}$.

Условие 2 представляет собой условие дифференцируемости (по Фреше) маргинальной плотности в пространстве $L_{1}(\mu)$ интегрируемых относительно меры $\mu$ функций (см. похожие условия в [15] и в [14]).

Нетрудно видеть, что условие 2 гарантирует дифференцируемость функции мощности любого критерия, основанного на фиксированном числе наблюдений, и возможность ее дифференцирования под знаком интеграла. Таким образом, в случае независимых одинаково распределенных наблюдений условие 2 влечет выполнение предположения 3 в [8].

У с л о в и е 3 . Существует $0<\gamma_{2}<\infty$ такое, что

$$
\mathbf{E}_{\theta_{0}}\left|\frac{\dot{f}_{\theta_{0}, j}\left(X_{j}\right)}{f_{\theta_{0}, j}\left(X_{j}\right)}\right| \leqslant \gamma_{2}
$$

для всех $j=1,2, \ldots$.

Здесь и далее математическое ожидание относительно любой «функции плотности» распределения $f(x)$

$$
\mathbf{E} g(X)=\int g(x) f(x) d \mu(x)
$$

понимается как

$$
\mathbf{E} g(X)=\int g(x) f(x) I_{\{f(x) \neq 0\}} d \mu(x),
$$

благодаря чему можно не заботиться об определении $g(x)$ на $\{f(x)=0\}$. 
Условие 3 является более слабым, чем предположение 4 в [8] для независимых одинаково распределенных наблюдений, в котором требуется конечность информации Фишера. В частности, если информация Фишера равномерно (по всем наблюдениям) ограничена:

$$
I_{j}\left(\theta_{0}\right)=\mathbf{E}_{\theta_{0}}\left(\frac{\dot{f}_{\theta_{0}, j}\left(X_{j}\right)}{f_{\theta_{0}, j}\left(X_{j}\right)}\right)^{2} \leqslant \gamma_{2}^{2}
$$

для всех $j=1,2, \ldots$, то из неравенства Гёльдера следует, что условие 3 выполнено. В свою очередь, это последнее условие тесно связано с условием 1 , поскольку при достаточно общих предположениях регулярности статистического эксперимента справедливо соотношение

$$
I_{j}(\theta, \theta+h) \sim \frac{1}{2} I_{j}(\theta) h^{2}, \quad h \rightarrow 0 .
$$

В случае независимых одинаково распределенных наблюдений условие 3 является следствием условия 2 , которое гарантирует существование конечного $\mathbf{E}_{\theta_{0}}\left|\dot{f}_{\theta_{0}, j}\left(X_{j}\right) / f_{\theta_{0}, j}\left(X_{j}\right)\right|$. Более общим образом, нетрудно видеть, что если «о-малое» в условии 2 является равномерным по $j$, то условие 3 вытекает из условий 1-2 в силу неравенства Пинскера (см. [5]).

Поскольку выражение типа $\left(1-\psi_{1}\right) \cdots\left(1-\psi_{n-1}\right) \psi_{n}$ будет встречаться очень часто (см., например, $(1),(2))$, введем для него следующее обозначение:

$$
s_{n}^{\psi}=\left(1-\psi_{1}\right) \cdots\left(1-\psi_{n-1}\right) \psi_{n}, \quad n=1,2, \ldots .
$$

Обозначим также

$$
t_{n}^{\psi}=\left(1-\psi_{1}\right) \cdots\left(1-\psi_{n-1}\right), \quad n=1,2, \ldots
$$

$\left(s_{1}^{\psi} \equiv \psi_{1}\right.$ и $t_{1}^{\psi} \equiv 1$ по определению).

Пусть, наконец,

$$
\begin{aligned}
& S_{n}^{\psi}=\left\{\left(x_{1}, \ldots, x_{n}\right): s_{n}^{\psi}\left(x_{1}, \ldots, x_{n}\right)>0\right\} \\
& T_{n}^{\psi}=\left\{\left(x_{1}, \ldots, x_{n}\right): t_{n}^{\psi}\left(x_{1}, \ldots, x_{n}\right)>0\right\} .
\end{aligned}
$$

3. Дифференцируемость функции мощности и информационные неравенства для характеристик критериев. В этом пункте мы докажем существование производной от функции мощности любого критерия с конечным, при нулевой гипотезе, средним объемом наблюдений и выведем неравенства, связывающие эту производную с другими характеристиками критерия: средним объемом выборки и вероятностью ошибки первого рода. 
Определим информацию Кульбака-Лейблера, содержащуюся в наблюдениях процесса $X_{1}, \ldots, X_{n}, \ldots$ до случайного момента остановки, определяемого правилом $\psi$, как

$$
I\left(\theta_{0}, \theta ; \psi\right)=\sum_{n=1}^{\infty} \mathbf{E}_{\theta_{0}} s_{n}^{\psi}\left(\sum_{j=1}^{n} \ln \frac{f_{\theta_{0}, j}}{f_{\theta, j}}\right)
$$

(отметим, что случайный процесс наблюдений $X_{1}, X_{2}, \ldots$ участвует в определении (5) неявным образом, через посредство $s_{n}^{\psi}=s_{n}^{\psi}\left(X_{1}, \ldots, X_{n}\right)$ и $f_{\theta, j}=f_{\theta, j}\left(X_{j}\right)$, так же как и в определении информации в одном наблюдении в (3)).

Следующие две леммы будут полезны для оценок, связанных с информационным количеством Кульбака-Лейблера.

Первая из них фактически является вариантом неравенства Йенсена, приспособленным к последовательным экспериментам.

Лемма 1. Пусть $G:[0, \infty) \rightarrow \mathbf{R} \cup\{\infty\}-$ любая выпукклая функuия, и пусть $a_{n}=a_{n}\left(x_{1}, \ldots, x_{n}\right), b_{n}=b_{n}\left(x_{1}, \ldots, x_{n}\right), n=1,2, \ldots,-$ любые последовательности неотрицательных измеримых функиий. Тогда, если

$$
0<\sum_{n=1}^{\infty} \mathbf{E}_{\theta_{0}} s_{n}^{\psi} a_{n}<\infty
$$

mo

$$
\frac{\sum_{n=1}^{\infty} \mathbf{E}_{\theta_{0}} s_{n}^{\psi} a_{n} G\left(b_{n}\right)}{\sum_{n=1}^{\infty} \mathbf{E}_{\theta_{0}} s_{n}^{\psi} a_{n}} \geqslant G\left(\frac{\sum_{n=1}^{\infty} \mathbf{E}_{\theta_{0}} s_{n}^{\psi} a_{n} b_{n}}{\sum_{n=1}^{\infty} \mathbf{E}_{\theta_{0}} s_{n}^{\psi} a_{n}}\right) .
$$

В частности, применяя лемму 1 к $G(x)=-\ln x, a_{n} \equiv 1, b_{n}=f_{\theta}^{n} / f_{\theta_{0}}^{n}$ и предполагая, что $\mathbf{P}_{\theta_{0}}\left(\tau_{\psi}<\infty\right)=\sum_{n=1}^{\infty} \mathbf{E}_{\theta_{0}} s_{n}^{\psi}=1$, получаем, что

$$
I\left(\theta_{0}, \theta ; \psi\right) \geqslant-\ln \left(\sum_{n=1}^{\infty} \mathbf{E}_{\theta} s_{n}^{\psi}\right) \geqslant 0 .
$$

Пусть теперь $(\psi, \phi)$ - любой последовательный критерий с $\mathbf{P}_{\theta_{0}}\left(\tau_{\psi}<\right.$ $\infty)=1$. Предположим, что $0<\beta_{\theta_{0}}(\psi, \phi)<1$. Тогда

$$
\begin{aligned}
I\left(\theta_{0}, \theta ; \psi\right)= & \beta_{\theta_{0}}(\psi, \phi) \frac{\sum_{n=1}^{\infty} \mathbf{E}_{\theta_{0}} s_{n}^{\psi} \phi_{n}\left(-\ln b_{n}\right)}{\beta_{\theta_{0}}(\psi, \phi)} \\
& +\left(1-\beta_{\theta_{0}}(\psi, \phi)\right) \frac{\sum_{n=1}^{\infty} \mathbf{E}_{\theta_{0}} s_{n}^{\psi}\left(1-\phi_{n}\right)\left(-\ln b_{n}\right)}{1-\beta_{\theta_{0}}(\psi, \phi)}
\end{aligned}
$$

где по-прежнему $b_{n}=f_{\theta}^{n} / f_{\theta_{0}}^{n}$. Поэтому, применяя лемму 1 к каждой из дробей в правой части $(6)$, получаем

$$
I\left(\theta_{0}, \theta ; \psi\right) \geqslant-\beta_{\theta_{0}}(\psi, \phi) \ln \frac{\sum_{n=1}^{\infty} \mathbf{E}_{\theta_{0}} s_{n}^{\psi} \phi_{n} b_{n}}{\beta_{\theta_{0}}(\psi, \phi)}
$$




$$
\begin{aligned}
& -\left(1-\beta_{\theta_{0}}(\psi, \phi)\right) \ln \frac{\sum_{n=1}^{\infty} \mathbf{E}_{\theta_{0}} s_{n}^{\psi}\left(1-\phi_{n}\right) b_{n}}{1-\beta_{\theta_{0}}(\psi, \phi)} \\
\geqslant & -\beta_{\theta_{0}}(\psi, \phi) \ln \frac{\sum_{n=1}^{\infty} \mathbf{E}_{\theta} s_{n}^{\psi} \phi_{n}}{\beta_{\theta_{0}}(\psi, \phi)} \\
& -\left(1-\beta_{\theta_{0}}(\psi, \phi)\right) \ln \frac{\sum_{n=1}^{\infty} \mathbf{E}_{\theta} s_{n}^{\psi}\left(1-\phi_{n}\right)}{1-\beta_{\theta_{0}}(\psi, \phi)} \\
\geqslant & -\beta_{\theta_{0}}(\psi, \phi) \ln \frac{\beta_{\theta}(\psi, \phi)}{\beta_{\theta_{0}}(\psi, \phi)}-\left(1-\beta_{\theta_{0}}(\psi, \phi)\right) \ln \frac{1-\beta_{\theta}(\psi, \phi)}{1-\beta_{\theta_{0}}(\psi, \phi)},
\end{aligned}
$$

т.e.

$$
I\left(\theta_{0}, \theta ; \psi\right) \geqslant \beta_{\theta_{0}}(\psi, \phi) \ln \frac{\beta_{\theta_{0}}(\psi, \phi)}{\beta_{\theta}(\psi, \phi)}+\left(1-\beta_{\theta_{0}}(\psi, \phi)\right) \ln \frac{1-\beta_{\theta_{0}}(\psi, \phi)}{1-\beta_{\theta}(\psi, \phi)}
$$

(более общие неравенства информационного типа можно найти в [1], см., например, лемму 5.1).

Точно так же получаем, что если $\beta_{\theta_{0}}(\psi, \phi)=0$, то

$$
I\left(\theta_{0}, \theta ; \psi\right) \geqslant-\ln \left(1-\beta_{\theta}(\psi, \phi)\right),
$$

и если $\beta_{\theta_{0}}(\psi, \phi)=1$, то

$$
I\left(\theta_{0}, \theta ; \psi\right) \geqslant-\ln \beta_{\theta}(\psi, \phi) .
$$

Следующая лемма (тождество Вальда для разнораспределенных слагаемых) полезна, в частности, для оценки информации в левой части (7).

Лемма 2. Пусть $Y_{j}=Y_{j}\left(X_{j}\right)$ - неотричательные измеримые функиии от наблюдений $X_{j}$ такие, что $\mathbf{E}_{\theta} Y_{j}<\infty, j=1,2, \ldots$ Тогда для любого правила остановки $\psi$ такого, ито $\mathbf{P}_{\theta}\left(\tau_{\psi}<\infty\right)=1$, выполнено равенство

$$
\sum_{n=1}^{\infty} \mathbf{E}_{\theta} s_{n}^{\psi}\left(\sum_{j=1}^{n} Y_{j}\right)=\sum_{j=1}^{\infty} \mathbf{E}_{\theta} Y_{j} \mathbf{P}_{\theta}\left(\tau_{\psi} \geqslant j\right) .
$$

Д о к а з а т е л ь с т в о. Пусть, для краткости, $\mathbf{E}$ и $\mathbf{P}$ обозначают соответственно $\mathbf{E}_{\theta}$ и $\mathbf{P}_{\theta}$ на протяжении всего доказательства.

Предположим, что левая часть (10) конечна. Тогда

$$
\sum_{n=1}^{\infty} \mathbf{E} s_{n}^{\psi}\left(\sum_{j=1}^{n} Y_{j}\right)=\sum_{n=1}^{\infty} \sum_{j=1}^{n} \mathbf{E} s_{n}^{\psi} Y_{j}=\sum_{j=1}^{\infty} \sum_{n=j}^{\infty} \mathbf{E} s_{n}^{\psi} Y_{j}
$$

(возможность изменения порядка суммирования обеспечивается конечностью исходного ряда). Нетрудно видеть, что при выполнении условий леммы

$$
\sum_{n=j}^{\infty} \mathbf{E} s_{n}^{\psi} Y_{j}=\mathbf{E} t_{j}^{\psi} Y_{j} .
$$


При этом в силу независимости $t_{j}^{\psi}($ см. $(4))$ и $Y_{j}$ имеем

$$
\mathbf{E} t_{j}^{\psi} Y_{j}=\mathbf{E} t_{j}^{\psi} \mathbf{E} Y_{j}=\mathbf{E} Y_{j} \mathbf{P}\left(\tau_{\psi} \geqslant j\right)
$$

так что

$$
\sum_{n=1}^{\infty} \mathbf{E} s_{n}^{\psi}\left(\sum_{j=1}^{n} Y_{j}\right)=\sum_{j=1}^{\infty} \mathbf{E} Y_{j} \mathbf{P}\left(\tau_{\psi} \geqslant j\right) .
$$

Обращая эти рассуждения в предположении конечности правой части (10), убеждаемся, что равенство в (10) также имеет место. Лемма доказана.

Следствие 1. Предположим, что $I_{j}\left(\theta_{0}, \theta\right)<\gamma<\infty$ для любого $j=1,2, \ldots$. Тогда для любого правила остановки $\psi$ такого, что $\mathbf{E}_{\theta_{0}} \tau_{\psi}<\infty$, вьполнено равенство

$$
I\left(\theta_{0}, \theta ; \psi\right)=\sum_{j=1}^{\infty} I_{j}\left(\theta_{0}, \theta\right) \mathbf{P}_{\theta_{0}}\left(\tau_{\psi} \geqslant j\right)
$$

Док аз а т ель с тв о. Пусть

$$
Y_{j}=\ln \frac{f_{\theta_{0}, j}}{f_{\theta, j}}, \quad Y_{j}^{+}=\max \left\{0, Y_{j}\right\}, \quad Y_{j}^{-}=\max \left\{0,-Y_{j}\right\} .
$$

Поскольку

$$
\begin{aligned}
\mathbf{E}_{\theta_{0}} Y_{j}^{-} & =\mathbf{E}_{\theta_{0}} \max \left\{0, \ln \frac{f_{\theta, j}}{f_{\theta_{0}, j}}\right\} \leqslant \mathbf{E}_{\theta_{0}} \max \left\{0, \frac{f_{\theta, j}}{f_{\theta_{0}, j}}-1\right\} \\
& \leqslant \int\left|f_{\theta, j}-f_{\theta_{0}, j}\right| d \mu \leqslant 2,
\end{aligned}
$$

то из леммы 2 получаем

$$
\sum_{n=1}^{\infty} \mathbf{E}_{\theta_{0}} s_{n}^{\psi}\left(\sum_{j=1}^{n} Y_{j}^{-}\right)=\sum_{j=1}^{\infty} \mathbf{E}_{\theta_{0}} Y_{j}^{-} \mathbf{P}_{\theta_{0}}\left(\tau_{\psi} \geqslant j\right),
$$

причем правая часть (12) конечна, поскольку $\sum_{j=1}^{\infty} \mathbf{P}_{\theta_{0}}\left(\tau_{\psi} \geqslant j\right)=\mathbf{E}_{\theta_{0}} \tau_{\psi}$. Теперь из условия $I_{j}\left(\theta_{0}, \theta\right)<\gamma, j \geqslant 1$, следует, что $\mathbf{E}_{\theta_{0}} Y_{j}^{+}<\gamma+2, j \geqslant 1$, так что из леммы 2 получаем

$$
\sum_{n=1}^{\infty} \mathbf{E}_{\theta_{0}} s_{n}^{\psi}\left(\sum_{j=1}^{n} Y_{j}^{+}\right)=\sum_{j=1}^{\infty} \mathbf{E}_{\theta_{0}} Y_{j}^{+} \mathbf{P}_{\theta_{0}}\left(\tau_{\psi} \geqslant j\right),
$$

и правая часть (13) также конечна. Благодаря этому, вычитая левую и правую части (12) из соответствующих частей (13) и применяя затем вычитание почленно, получаем (11). Следствие доказано. 
Поскольку $\sum_{j=1}^{\infty} \mathbf{P}\left(\tau_{\psi} \geqslant j\right)=\mathbf{E} \tau_{\psi}$, то при выполнении условия 1 из леммы 2 следует, что

$$
I\left(\theta_{0}, \theta ; \psi\right) \leqslant \gamma_{1}\left(\theta-\theta_{0}\right)^{2} \mathbf{E}_{\theta_{0}} \tau_{\psi}
$$

если $\left|\theta-\theta_{0}\right| \leqslant \delta$.

Следующая теорема, являющаяся следствием информационного неравенства (7), представляет самостоятельный интерес, поскольку дает границы для характеристик (среднего объема выборки, вероятности ошибки первого рода и производной функции мощности) любого критерия проверки гипотез.

Теорема 1. Предположим, ито выполнено условие 1. Тогда для любого последовательного критерия $(\psi, \phi)$ такого, ито $\mathbf{E}_{\theta_{0}} \tau_{\psi}<\infty u$ производная $\dot{\beta}_{\theta_{0}}(\psi, \phi)$ функиии мощности $\beta_{\theta}(\psi, \phi)$ в точке $\theta=\theta_{0}$ существует,

$$
\left(\dot{\beta}_{\theta_{0}}(\psi, \phi)\right)^{2} \leqslant 2 \gamma_{1} \beta_{\theta_{0}}(\psi, \phi)\left(1-\beta_{\theta_{0}}(\psi, \phi)\right) \mathbf{E}_{\theta_{0}} \tau_{\psi} .
$$

Д о к а з а т е л ь с т в о. Поскольку на всем протяжении этого доказательства последовательный критерий $(\psi, \phi)$ остается фиксированным, будем обозначать просто $\beta_{h}=\beta_{\theta_{0}+h}(\psi, \phi)$ для любого $h$ и $\dot{\beta}_{0}=$ $\left.\left(\beta_{\theta}(\psi, \phi)\right)_{\theta}^{\prime}\right|_{\theta=\theta_{0}}$, предполагая при этом, что для $(\psi, \phi)$ выполнены условия теоремы 1. Аналогичным образом «перепараметризуем» семейство плотностей, и будем писать $f_{h, j}$ вместо $f_{\theta_{0}+h, j}, I_{j}(0, h)$ вместо $I_{j}\left(\theta_{0}, \theta_{0}+h\right), I(0, h ; \psi)$ вместо $I\left(\theta_{0}, \theta_{0}+h ; \psi\right)$ и просто $\mathbf{E}$ и $\mathbf{P}$ вместо $\mathbf{E}_{\theta_{0}}$ и $\mathbf{P}_{\theta_{0}}$ соответственно.

Выведем теперь из соотношения (14), что выполнено неравенство $\left(\dot{\beta}_{0}\right)^{2} \leqslant 2 \gamma_{1} \beta_{0}\left(1-\beta_{0}\right) \mathbf{E} \tau_{\psi}$, т.е. (15).

Пусть сначала $0<\beta_{0}<1$. Обозначим

$$
w(x)=\beta_{0} \ln \frac{\beta_{0}}{x}+\left(1-\beta_{0}\right) \ln \frac{1-\beta_{0}}{1-x},
$$

где $x \in[0,1]$ (см. правую часть неравенства (7)). Из (7) и (14) следует, что

$$
0 \leqslant w\left(\beta_{h}\right) \leqslant \gamma_{1} h^{2} \mathbf{E} \tau_{\psi}
$$

поэтому очевидно, что $\beta_{h} \rightarrow \beta_{0}, h \rightarrow 0$.

Пусть $\Delta_{h} \beta=\beta_{h}-\beta_{0}$. Тогда по формуле Тейлора для $\ln (1+x)$

$$
\begin{aligned}
w\left(\beta_{h}\right) & =-\beta_{0} \ln \left(1+\frac{\Delta_{h} \beta}{\beta_{0}}\right)-\left(1-\beta_{0}\right) \ln \left(1-\frac{\Delta_{h} \beta}{1-\beta_{0}}\right) \\
& =\frac{\left(\Delta_{h} \beta\right)^{2}}{2 \beta_{0}}+\frac{\left(\Delta_{h} \beta\right)^{2}}{2\left(1-\beta_{0}\right)}+o\left(\left(\Delta_{h} \beta\right)^{2}\right) \\
& =\frac{\left(\Delta_{h} \beta\right)^{2}}{2 \beta_{0}\left(1-\beta_{0}\right)}+o\left(\left(\Delta_{h} \beta\right)^{2}\right), \quad h \rightarrow 0,
\end{aligned}
$$


откуда в силу (16) следует

$$
\frac{\left(\Delta_{h} \beta / h\right)^{2}}{2 \beta_{0}\left(1-\beta_{0}\right)}+o\left(\left(\frac{\Delta_{h} \beta}{h}\right)^{2}\right) \leqslant \gamma_{1} \mathbf{E} \tau_{\psi}, \quad h \rightarrow 0,
$$

т.е. $\left(\dot{\beta}_{0}\right)^{2} /\left(2 \beta_{0}\left(1-\beta_{0}\right)\right) \leqslant \gamma_{1} \mathbf{E} \tau_{\psi}$, что эквивалентно (15).

Пусть теперь $\beta_{0}=0$. Из (8) и (14) следует, что $\Delta_{h} \beta / h \rightarrow 0$, когда $h \rightarrow 0$, т.е. $\dot{\beta}_{0}=0$. Таким образом, (15) также выполнено.

Если $\beta_{0}=1$, то аналогичным образом из (9) получаем, что $\dot{\beta}_{0}=0$. Теорема доказана.

3 а м е ч а н и е 1. В случае независимых одинаково распределенных наблюдений из регулярного семейства распределений из доказательства теоремы 1 легко видеть, что

$$
\left(\dot{\beta}_{\theta_{0}}(\psi, \phi)\right)^{2} \leqslant \beta_{\theta_{0}}(\psi, \phi)\left(1-\beta_{\theta_{0}}(\psi, \phi)\right) I\left(\theta_{0}\right) \mathbf{E}_{\theta_{0}} \tau_{\psi},
$$

где $I\left(\theta_{0}\right)$ - информационное количество Фишера. По-видимому, это неравенство справедливо и для широкого класса процессов с непрерывным временем (например, рассмотренного, в связи с локально наиболее мощными критериями, в [22] класса процессов с независимыми стационарными приращениями). Любопытно отметить, что в этом случае в неравенстве (17) достигается равенство для локально наиболее мощного критерия проверки гипотез о винеровском процессе с линейным сносом (см. [15]). В частности, из (17) следует оптимальность этого критерия в более широком, чем в [22], классе критериев, а именно в классе критериев с вероятностью ошибки, меньшей или равной заданной $\alpha<0.5$, и со средним объемом выборки, не превосходящим заданного (см. [21] в связи с расширенным понятием оптимальности для процессов с дискретным временем).

Теорема 2. Пусть выполнены условия 1-3. Тогда функиия мощности любого критерия $(\psi, \phi)$, для которого $\mathbf{E}_{\theta_{0}} \tau_{\psi}<\infty$, дифференцируема в $\theta=\theta_{0}$ u

$$
\dot{\beta}_{\theta_{0}}(\psi, \phi)=\sum_{n=1}^{\infty} \mathbf{E}_{\theta_{0}}\left(s_{n}^{\psi} \phi_{n} \sum_{j=1}^{n} q_{j}\right)
$$

гдe

$$
q_{n}=q_{n}\left(x_{n}\right)=\frac{\dot{f}_{\theta_{0}, n}\left(x_{n}\right)}{f_{\theta_{0}, n}\left(x_{n}\right)} .
$$

Д о к а з а т е л ь с т в о. Пусть $(\psi, \phi)$ - любой критерий с $\mathbf{E}_{\theta_{0}} \tau_{\psi}<$ $\infty$. Докажем, что

$$
\frac{\beta_{\theta}(\psi, \phi)-\beta_{\theta_{0}}(\psi, \phi)}{\theta-\theta_{0}}-\sum_{n=1}^{\infty} \mathbf{E}_{\theta_{0}}\left(s_{n}^{\psi} \phi_{n} \sum_{j=1}^{n} q_{j}\right) \rightarrow 0, \quad \theta \rightarrow \theta_{0},
$$


т.e.

$$
\sum_{n=1}^{\infty} \int s_{n}^{\psi} \phi_{n}\left(\frac{f_{\theta}^{n}-f_{\theta_{0}}^{n}}{\theta-\theta_{0}}-\dot{f}_{\theta_{0}}^{n}\right) d \mu^{n} \rightarrow 0, \quad \theta \rightarrow \theta_{0},
$$

где $\dot{f}_{\theta_{0}}^{n}=\left(\sum_{j=1}^{n} q_{j}\right) f_{\theta_{0}}^{n}$ (нетрудно видеть, что

$$
\mathbf{E}_{\theta_{0}}\left(s_{n}^{\psi} \phi_{n} \sum_{j=1}^{n} q_{j}\right)=\int s_{n}^{\psi} \phi_{n} \dot{f}_{\theta_{0}}^{n} d \mu^{n},
$$

поскольку из условия 2 следует, что $\dot{f}_{\theta_{0}, j}=0 \mu$-почти наверное на $\left.\left\{x: f_{\theta_{0}, j}(x)=0\right\}\right)$.

Из условия 2 нетрудно извлечь, что для любого фиксированного $k \geqslant 1$

$$
\sum_{n=1}^{k} \int s_{n}^{\psi} \phi_{n}\left(\frac{f_{\theta}^{n}-f_{\theta_{0}}^{n}}{\theta-\theta_{0}}-\dot{f}_{\theta_{0}}^{n}\right) d \mu^{n} \rightarrow 0, \quad \theta \rightarrow \theta_{0}
$$

(по-существу, это - дифференцируемость произведения $f_{\theta}^{n}=\prod_{j=1}^{n} f_{\theta, j}$ в $L_{1}\left(\mu^{n}\right)$ в предположении дифференцируемости сомножителей $f_{\theta, j}$ в $\left.L_{1}(\mu)\right)$. Поэтому чтобы доказать $(18)$, достаточно показать, что для любого $\varepsilon>0$ существует такое $k>1$, что

$$
\limsup _{\theta \rightarrow \theta_{0}}\left|\sum_{n=k}^{\infty} \int s_{n}^{\psi} \phi_{n}\left(\frac{f_{\theta}^{n}-f_{\theta_{0}}^{n}}{\theta-\theta_{0}}-\dot{f}_{\theta_{0}}^{n}\right) d \mu^{n}\right|<2 \varepsilon .
$$

Очевидно, для доказательства (19) достаточно показать, что можно найти такое $k$, что

$$
\limsup _{\theta \rightarrow \theta_{0}}\left|\sum_{n=k}^{\infty} \int s_{n}^{\psi} \phi_{n} \frac{f_{\theta}^{n}-f_{\theta_{0}}^{n}}{\theta-\theta_{0}} d \mu^{n}\right|<\varepsilon
$$

и

$$
\sum_{n=k}^{\infty} \int s_{n}^{\psi}\left|\dot{f}_{\theta_{0}}^{n}\right| d \mu^{n}=\sum_{n=k}^{\infty} \mathbf{E}_{\theta_{0}}\left(s_{n}^{\psi}\left|\sum_{j=1}^{n} q_{j}\right|\right)<\varepsilon .
$$

Обратимся сначала к доказательству (21). Для этого заметим, что, согласно лемме 2 ,

$$
\sum_{n=1}^{\infty} \mathbf{E}_{\theta_{0}}\left(s_{n}^{\psi} \sum_{j=1}^{n}\left|q_{j}\right|\right)=\sum_{j=1}^{\infty} \mathbf{E}_{\theta_{0}}\left|q_{j}\right| \mathbf{P}_{\theta_{0}}\left(\tau_{\psi} \geqslant j\right),
$$

где ряд в правой части конечен, поскольку из условия 3 вытекает, что $\mathbf{E}_{\theta_{0}}\left|q_{j}\right| \leqslant \gamma_{2}<\infty$.

Следовательно, ряд в левой части (22) сходится, откуда следует (21). 
Докажем теперь, что существует такое $k$, что выполняется (20). Для этого применим лемму 1 с $G(x)=-\ln x, a_{n}=\phi_{n} I_{\{n \geqslant k\}}, b_{n}=f_{\theta}^{n} / f_{\theta_{0}}^{n}$.

Пусть для краткости

$$
\alpha_{k}=\sum_{n=k}^{\infty} \mathbf{E}_{\theta_{0}} s_{n}^{\psi} \phi_{n}, \quad \alpha_{k}(\theta)=\sum_{n=k}^{\infty} \mathbf{E}_{\theta} s_{n}^{\psi} \phi_{n}
$$

и предположим сначала, что $0<\alpha_{k}<1$. Тогда

$$
\begin{aligned}
I\left(\theta_{0}, \theta ; \psi\right)= & \alpha_{k} \frac{\sum_{n=1}^{\infty} \mathbf{E}_{\theta_{0}} s_{n}^{\psi} \phi_{n} I_{\{n \geqslant k\}}\left(-\ln b_{n}\right)}{\alpha_{k}} \\
& +\left(1-\alpha_{k}\right) \frac{\sum_{n=1}^{\infty} \mathbf{E}_{\theta_{0}} s_{n}^{\psi}\left(1-\phi_{n} I_{\{n \geqslant k\}}\right)\left(-\ln b_{n}\right)}{1-\alpha_{k}} .
\end{aligned}
$$

Применяя к каждой из дробей в правой части (23) лемму 1 (как при выводе (7)), получаем

$$
\begin{aligned}
I\left(\theta_{0}, \theta ; \psi\right) & \geqslant-\alpha_{k} \ln \frac{\alpha_{k}(\theta)}{\alpha_{k}}-\left(1-\alpha_{k}\right) \ln \frac{1-\alpha_{k}(\theta)}{1-\alpha_{k}} \\
& =-\alpha_{k} \ln \left(1+\frac{\alpha_{k}(\theta)-\alpha_{k}}{\alpha_{k}}\right)-\left(1-\alpha_{k}\right) \ln \left(1-\frac{\alpha_{k}(\theta)-\alpha_{k}}{1-\alpha_{k}}\right) .
\end{aligned}
$$

Поскольку, согласно (14), левая часть (24) стремится к нулю при $\theta \rightarrow \theta_{0}$, то, вполне аналогично доказательству теоремы 1 , сначала получаем, что $\alpha_{k}(\theta) \rightarrow \alpha_{k}$ при $\theta \rightarrow \theta_{0}$, а затем, применяя формулу Тейлора для $\ln (1+x)$ в точке $x=0$ до членов второго порядка, получаем неравенство

$$
\frac{\left(\alpha_{k}(\theta)-\alpha_{k}\right)^{2}}{2 \alpha_{k}\left(1-\alpha_{k}\right)}+o\left(\left(\alpha_{k}(\theta)-\alpha_{k}\right)^{2}\right) \leqslant \gamma_{1}\left(\theta-\theta_{0}\right)^{2} .
$$

Следовательно,

$$
\limsup _{\theta \rightarrow \theta_{0}}\left|\frac{\alpha_{k}(\theta)-\alpha_{k}}{\theta-\theta_{0}}\right| \leqslant \sqrt{2 \gamma_{1} \alpha_{k}} \leqslant \sqrt{2 \gamma_{1} \mathbf{P}_{\theta_{0}}\left(\tau_{\psi} \geqslant k\right)} .
$$

Таким образом, (20) выполняется, если $\sqrt{2 \gamma_{1} \mathbf{P}_{\theta_{0}}\left(\tau_{\psi} \geqslant k\right)} \leqslant \varepsilon$, что можно обеспечить, поскольку, по условию, $\mathbf{E}_{\theta_{0}} \tau_{\psi}<\infty$.

Рассмотрим теперь случай $\alpha_{k}=\sum_{n \geqslant k} \mathbf{E}_{\theta_{0}} s_{n}^{\psi} \phi_{n}=0$. Согласно лемме 1 ,

$$
\begin{aligned}
I\left(\theta_{0}, \theta ; \psi\right) & =\sum_{n=1}^{\infty} \mathbf{E}_{\theta_{0}} s_{n}^{\psi}\left(-\ln \frac{f_{\theta}^{n}}{f_{\theta_{0}}^{n}}\right)\left(1-\phi_{n} I_{\{n \geqslant k\}}\right) \\
& \geqslant-\ln \left(\sum_{n=1}^{\infty} \mathbf{E}_{\theta_{0}} s_{n}^{\psi} \frac{f_{\theta}^{n}}{f_{\theta_{0}}^{n}}\left(1-\phi_{n} I_{\{n \geqslant k\}}\right)\right) \\
& \geqslant-\ln \left(1-\sum_{n=k}^{\infty} \mathbf{E}_{\theta} \phi_{n} I_{\{n \geqslant k\}}\right) \\
& =-\ln \left(1-\alpha_{k}(\theta)\right) \geqslant \alpha_{k}(\theta)=\alpha_{k}(\theta)-\alpha_{k} .
\end{aligned}
$$


В силу (14) отсюда следует, что

$$
\lim _{\theta \rightarrow \theta_{0}} \frac{\alpha_{k}(\theta)-\alpha_{k}}{\left|\theta-\theta_{0}\right|}=0
$$

т.е. в этом случае (20) также выполняется.

Аналогично доказывается, что если $\alpha_{k}=1$, то

$$
\lim _{\theta \rightarrow \theta_{0}} \frac{1-\alpha_{k}(\theta)}{\left|\theta-\theta_{0}\right|}=0
$$

т.е. (20) также выполняется. Теорема доказана.

3 а м е ч а н и е 2. Теорема 2 представляет собой обобщение, на случай разнораспределенных наблюдений и рандомизированных правил остановки и принятия решения, леммы 4.1.4 [11]. Для независимых одинаково распределенных наблюдений этот результат был впервые анонсирован в [8] и восходит к неопубликованной работе [7]. Доказательство этого результата в [11] следует работе [13]. Сходные вопросы об условиях существования вторых производных от функции мощности последовательных критериев, по-видимому, до настоящего времени остаются без ответа (см. [14]).

4. Структура оптимальных последовательных критериев. Усеченные правила остановки. В этом пункте мы даем характеризацию оптимальных усеченных последовательных критериев.

Для любого натурального $N$ обозначим через $\mathscr{F}^{N}$ класс усеченных (до этапа $N$ ) правил остановки, т.е. таких $\psi$, для которых $\left(1-\psi_{1}\right)(1-$ $\left.\psi_{2}\right) \cdots\left(1-\psi_{N}\right) \equiv 1$.

Начнем построение оптимальных усеченных правил с определения следующих функций.

Пусть $g(z)=\min \{0, z\}, z \in \mathbf{R}$. Определим для любых $N \geqslant 1$ и $n=1, \ldots, N$ функции $v_{n}^{N}(z)=v_{n}^{N}(z ; c), z \in \mathbf{R}$, начиная с

$$
v_{N}^{N}(z) \equiv g(z), \quad z \in \mathbf{R},
$$

с помощью рекуррентных соотношений

$$
v_{n-1}^{N}(z ; c)=\min \left\{g(z), c+\mathbf{E}_{\theta_{0}} v_{n}^{N}\left(z-q_{n} ; c\right)\right\},
$$

$n=N, N-1, \ldots, 1$, где, по определению, $q_{n}=q_{n}\left(x_{n}\right)=\dot{f}_{\theta_{0}, n}\left(x_{n}\right) / f_{\theta_{0}, n}\left(x_{n}\right)$. Пусть

$$
r_{n-1}^{N}(z)=r_{n-1}^{N}(z ; c)=\mathbf{E}_{\theta_{0}} v_{n}^{N}\left(z-q_{n} ; c\right),
$$

$n=1, \ldots, N$. 
Для произвольных $b \in \mathbf{R}$ и $c>0$ определим, следуя [21], «функцию множителей Лагранжа»

$$
L_{N}(\psi ; b, c)=\sum_{n=1}^{N} \mathbf{E}_{\theta_{0}} s_{n}^{\psi}\left(n c+\min \left\{0, b-\sum_{i=1}^{n} q_{i}\right\}\right)
$$

для любого $\psi \in \mathscr{F}^{N}$ (см. в $[21$, формула (4.2)]).

Пусть также

$$
z_{n}=z_{n}\left(x_{1}, \ldots, x_{n}\right)=\sum_{i=1}^{n} q_{i}\left(x_{i}\right)
$$

(если условие $\prod_{i=1}^{n} f_{\theta_{0}, i}\left(x_{i}\right)>0$ не выполняется, положим $z_{n}=0$ ).

Теорема 3. Предположим, ито выполнено условие 2.

Тогда для всех $\psi \in \mathscr{F}^{N}$

$$
L_{N}(\psi ; b, c) \geqslant c+r_{0}^{N}(b ; c) .
$$

При этом равенство в (28) достигается тогда и только тогда, когда

$$
I_{\left\{g\left(b-z_{n}\right)<c+r_{n}^{N}\left(b-z_{n} ; c\right)\right\}} \leqslant \psi_{n} \leqslant I_{\left\{g\left(b-z_{n}\right) \leqslant c+r_{n}^{N}\left(b-z_{n} ; c\right)\right\}}
$$

$\mu^{n}$-почти всюду на $T_{n}^{\psi} \cap\left\{f_{\theta_{0}}^{n}>0\right\}$ для каждого $n=1, \ldots, N-1$.

Д о к а з а т е л ь с т в о. Для доказательства достаточно выразить элементы оптимального правила остановки из следствия $4.1[21]\left(V_{n}^{N}\right.$ и $R_{n}^{N}$ ) через соответствующие функции $v_{n}^{N}$ и $r_{n}^{N}$. Покажем, что для любых $N=1,2, \ldots$ и любых $n \leqslant N$

$$
V_{n}^{N}=v_{n}^{N}\left(b-z_{n}\right) f_{\theta_{0}}^{n}
$$

$\mu^{n}$-почти всюду.

Проведем доказательство по индукции по $n=N, N-1, \ldots, 1$. Bce paвенства между функциями от наблюдений $\left(x_{1}, \ldots, x_{n}\right)$ будут пониматься $\mu^{n}$-почти всюду.

Для $n=N$, очевидно,

$$
V_{N}^{N}=l_{N}=\min \left\{0, b-z_{N}\right\} f_{\theta_{0}}^{N}=v_{N}^{N}\left(b-z_{N}\right) f_{\theta_{0}}^{N} .
$$

Предположим, что (30) выполняется для некоторого $n \leqslant N$. Тогда

$$
\begin{aligned}
V_{n-1}^{N} & =\min \left\{l_{n-1}, c f_{\theta_{0}}^{n-1}+\int V_{n}^{N} d \mu\left(x_{n}\right)\right\} \\
& =\min \left\{\min \left\{0, b f_{\theta_{0}}^{n-1}-\dot{f}_{\theta_{0}}^{n-1}\right\}, c f_{\theta_{0}}^{n-1}+\int v_{n}^{N}\left(b-z_{n}\right) f_{\theta_{0}}^{n} d \mu\left(x_{n}\right)\right\} \\
& =\min \left\{g\left(b-z_{n-1}\right), c+\int v_{n}^{N}\left(b-z_{n-1}-q_{n}\right) f_{\theta_{0}, n}\left(x_{n}\right) d \mu\left(x_{n}\right)\right\} f_{\theta_{0}}^{n-1} \\
& =v_{n-1}^{N}\left(b-z_{n-1}\right) f_{\theta_{0}}^{n-1} .
\end{aligned}
$$


Таким образом, (30) доказано.

Далее,

$$
\begin{aligned}
R_{n-1}^{N} & =\int V_{n} d \mu\left(x_{n}\right)=\int v_{n}^{N}\left(b-z_{n-1}-q_{n}\right) f_{\theta_{0}, n}\left(x_{n}\right) d \mu\left(x_{n}\right) f_{\theta_{0}}^{n-1} \\
& =r_{n-1}^{N}\left(b-z_{n-1}\right) f_{\theta_{0}}^{n-1}
\end{aligned}
$$

для любого $n=1, \ldots, N$.

Теперь очевидно, что (29) эквивалентно (4.5) в [21], если $f_{\theta_{0}}^{n}>0$. Теорема доказана.

Следствие 2. Предположим, что выполнено условие 2, и пусть $b>0$ - любое вещественное число.

Пусть $\psi \in \mathscr{F}^{N}-$ любое правило остановки, удовлетворяющее (29) $\mu^{n}$-почти всюду на $T_{n}^{\psi}$ для каждого $n=1, \ldots, N-1$, и пусть решающее правило ф таково, что

$$
I_{\left\{z_{n}>b\right\}} \leqslant \phi_{n} \leqslant I_{\left\{z_{n} \geqslant b\right\}}
$$

$\mu^{n}$-почти всюду на $S_{n}^{\psi}$ для любого $n=1, \ldots, N$.

Тогда критерий $(\psi, \phi)$ - локально наиболее мошный в классе всех (усеченных) критериев $\left(\psi^{\prime}, \phi^{\prime}\right)$ с $\psi^{\prime} \in \mathscr{F}^{N}$, в том смысле, что

$$
\dot{\beta}_{\theta_{0}}(\psi, \phi) \geqslant \dot{\beta}_{\theta_{0}}\left(\psi^{\prime}, \phi^{\prime}\right)
$$

если

$$
\alpha\left(\psi^{\prime}, \phi^{\prime}\right) \leqslant \alpha(\psi, \phi) \quad u \quad \mathscr{N}_{\theta_{0}}\left(\psi^{\prime}\right) \leqslant \mathscr{N}_{\theta_{0}}(\psi) .
$$

Неравенство (32) является строгим, если строгим является хотя бы одно из неравенств (33). Если во всех неравенствах в (32) и (33) достигается равенство, то $\psi^{\prime}$ также удовлетворяет (29) $\mu^{n}$-почти всюду на $T_{n}^{\psi^{\prime}}$ для каждого $n=1, \ldots, N-1\left(\right.$ с $\psi_{n}^{\prime}$ вместо $\left.\psi_{n}\right)$ и $\phi^{\prime}$ удовлетворяет (31) (с $\phi_{n}^{\prime}$ вместо $\left.\phi_{n}\right) \mu^{n}$-почти всюду на $S_{n}^{\psi^{\prime}}$ для любого $n=1, \ldots, N$.

Дальнейшую детализацию оптимальных правил остановки можно получить, изучая свойства всех задействованных в (29) функций. Сформулируем необходимые свойства в виде следующих лемм.

Лемма 3. Функции $v_{n}^{N}(z), n=0, \ldots, N, N=1,2, \ldots$, определяемье соотношениями (26), обладают следуюшими свойствами:

1) $v_{n}^{N}(z) \leqslant g(z), z \in \mathbf{R}$,

2) $v_{n}^{N}(z)$ - вогнутая и непрерывная функиия на $\mathbf{R}$,

3) $v_{n}^{N}(z)-$ неубывающая функиия на $\mathbf{R}$,

4) $z-v_{n}^{N}(z)-$ неубывающая функция на $\mathbf{R}$,

5) $g(z)-v_{n}^{N}(z) \rightarrow 0$ при $z \rightarrow \pm \infty$.

Д ок аз а тель с тво. В доказательстве будем использовать следующую элементарную лемму. 
Лемма 4. Пусть $F-$ вогнутая функиия на $\mathbf{R}$. Тогда для любого $n \geqslant 1$

$$
G_{n}(z)=\mathbf{E}_{\theta_{0}} F\left(z-q_{n}\right)
$$

- также вогнутая функиия от $z$. При этом $G_{n}(z) \leqslant F(z), z \in \mathbf{R}$.

Свойство 1) является прямым следствием определений (25) и (26).

Свойства 2)-5) докажем одновременно, пользуясь индукцией по $n=$ $N, N-1, \ldots, 1$.

Для $v_{N}^{N}(z) \equiv g(z)$ все упомянутые в 2)-5) свойства очевидны.

Предположим, что свойства 2)-5) имеют место для некоторого $n \leqslant$ $N$. Докажем, что тогда они выполняются для $v_{n-1}^{N}$.

Вследствие (26), $v_{n-1}^{N}$ - минимум двух вогнутых функций (вторая из них вогнута по лемме 4$)$. Таким образом, $v_{n-1}^{N}$ также вогнута.

Теперь непрерывность $v_{n-1}^{N}$ следует из теоремы 10.1 [6].

Если $v_{n}^{N}(z)$ не убывает, то по $(26) v_{n-1}^{N}(z)$ также не убывает. Поскольку $z-v_{n}^{N}(z)$ - неубывающая функция, то

$$
z-v_{n-1}^{N}(z)=\max \left\{\max \{0, z\},-c+\mathbf{E}_{\theta_{0}}\left(\left(z-q_{n}\right)-v_{n}^{N}\left(z-q_{n}\right)\right)\right\}
$$

— неубывающая, поскольку математическое ожидание в правой части неубывающая функция $z$.

Наконец, докажем, что $g(z)-v_{n-1}^{N}(z) \rightarrow 0$, когда $z \rightarrow \pm \infty$ (свойство 5) леммы).

Пусть сначала $z_{k}, k=1,2, \ldots$, - монотонно возрастающая последовательность, $z_{k} \rightarrow \infty, k \rightarrow \infty$.

Если $k$ достаточно велико, то $z_{k}>0$, поэтому $g\left(z_{k}\right)=0$ при таких $k$, так что

$$
g\left(z_{k}\right)-v_{n-1}^{N}\left(z_{k}\right)=-\min \left\{0, c+\mathbf{E}_{\theta_{0}} v_{n}^{N}\left(z_{k}-q_{n}\right)\right\} \rightarrow 0,
$$

когда $k \rightarrow \infty$, поскольку математическое ожидание сходится к нулю по теореме Лебега об ограниченной сходимости. Действительно, по предположению индукции $v_{n}^{N}\left(z_{k}-q_{n}\right) \rightarrow 0$, когда $k \rightarrow \infty$, и

$$
v_{n}^{N}\left(z_{1}-q_{n}\right) \leqslant v_{n}^{N}\left(z_{k}-q_{n}\right) \leqslant 0
$$

где функция $v_{n}^{N}\left(z_{1}-q_{n}\right)$ интегрируема, так как согласно свойствам 3 ) и 4) имеем:

$$
0 \leqslant g(z)-v_{n}^{N}(z) \leqslant-v_{n}^{N}(0)<\infty
$$

так что

$$
v_{n}^{N}\left(z_{1}-q_{n}\right) \geqslant g\left(z_{1}-q_{n}\right)+v_{n}^{N}(0),
$$

и при этом $\mathbf{E}_{\theta_{0}}\left|g\left(z_{1}-q_{n}\right)\right| \leqslant \mathbf{E}_{\theta_{0}}\left|z_{1}-q_{n}\right|<\infty$. 
Пусть теперь $z_{k}, k=1,2, \ldots,-$ монотонно убывающая последовательность, $z_{k} \rightarrow-\infty, k \rightarrow \infty$. При достаточно больших $k$ выполнено неравенство $z_{k}<0$, поэтому $g\left(z_{k}\right)=z_{k}$, и

$$
g\left(z_{k}\right)-v_{n-1}^{N}\left(z_{k}\right)=-\min \left\{0, c-\mathbf{E}_{\theta_{0}}\left(\left(z_{k}-q_{n}\right)-v_{n}^{N}\left(z_{k}-q_{n}\right)\right)\right\} \rightarrow 0
$$

при $z \rightarrow \infty$, поскольку математическое ожидание сходится к нулю по теореме Лебега об ограниченной сходимости. Действительно, $\left(z_{k}-q_{n}\right)-$ $v_{n}^{N}\left(z_{k}-q_{n}\right) \rightarrow 0$ при $k \rightarrow \infty$ согласно свойству 5), и при этом

$$
\left(z_{k}-q_{n}\right)-v_{n}^{N}\left(z_{k}-q_{n}\right) \leqslant\left(z_{1}-q_{n}\right)-v_{n}^{N}\left(z_{1}-q_{n}\right)
$$

согласно свойству 4), где функция в правой части неравенства интегрируема по тем же причинам, что и выше. Лемма доказана.

Лемма 5. Функции $r_{n}^{N}(z), n=0, \ldots, N, N=1,2, \ldots$, определяемые соотношениями (27), обладают следуюшими свойствами:

1) $r_{n}^{N}(z) \leqslant v_{n}^{N}(z), z \in \mathbf{R}$

2) $r_{n}^{N}(z)$ как функиия $z \in \mathbf{R}-$ вогнута и непрерьвна,

3) $r_{n}^{N}(z)$ как функиия $z \in \mathbf{R}$ является неубьвающей,

4) $z-r_{n}^{N}(z)$ как функиия $z \in \mathbf{R}$ является неубьвающей,

5) $g(z)-r_{n}^{N}(z) \rightarrow 0$ когда $z \rightarrow \pm \infty$.

Д о к а з а т е л ь с т в о. 1) Имеем по определению:

$$
\begin{aligned}
r_{n}^{N}(z)-v_{n}^{N}(z) & =-\min \left\{g(z)-r_{n}^{N}(z), c\right\} \\
& \leqslant-\min \left\{\mathbf{E}_{\theta_{0}}\left(g\left(z-q_{n}\right)-v_{n}^{N}\left(z-q_{n}\right)\right), c\right\} \leqslant 0,
\end{aligned}
$$

где первое неравенство следует из неравенства Йенсена, а второе - из свойства 1) леммы 3.

2) Согласно свойству 2$)$ леммы $3, v_{n+1}^{N}\left(z-q_{n+1}\right)$ - вогнутая функция $z$. Согласно лемме 4 , отсюда следует вогнутость $r_{n}^{N}$. Непрерывность $r_{n}^{N}$ следует теперь из теоремы 10.1 [6].

3) Согласно свойству 3$)$ леммы $3, v_{n+1}^{N}\left(z-q_{n+1}\right)$ - неубывающая функция $z$, отсюда следует, что $r_{n}^{N}(z)=\mathbf{E}_{\theta_{0}} v_{n+1}^{N}\left(z-q_{n+1}\right)-$ также неубывающая функция $z$.

4) Точно так же $z-r_{n}^{N}(z)=\mathbf{E}_{\theta_{0}}\left(\left(z-q_{n+1}\right)-v_{n+1}^{N}\left(z-q_{n+1}\right)\right)$ является неубывающей функцией $z$.

5) См. доказательство свойства 5) леммы 3.

Лемма доказана.

Лемма 6. Если $c+r_{n}^{N}(0) \leqslant 0$, то в каждой из областей $\{z \leqslant 0\}$ и $\{z \geqslant 0\}$ существует единственное решение уравнения

$$
c+r_{n}^{N}(z)=g(z) \text {. }
$$

Эти решения мьи обозначим $A_{n}^{N}=A_{n}^{N}(c) \leqslant 0$ и $B_{n}^{N}=B_{n}^{N}(c) \geqslant 0$. При этом $g(z)>c+r_{n}^{N}(z)$ тогда и только тогда, когда $A_{n}^{N}<z<B_{n}^{N}$.

Если с $+r_{n}^{N}(0)>0$, то уравнение (34) решения не имеет. 
Д о к а з а т е л ь с т в о. Функция $g(z)-r_{n}^{N}(z)$ непрерывна и является неотрицательной в силу свойства 1$)$ леммы 5 и свойства 1) леммы 3.

В силу свойств 3$)$ и 4) леммы 5 , функция $g(z)-r_{n}^{N}(z)$ - неубывающая при $z \leqslant 0$ и невозрастающая при $z \geqslant 0$. Поэтому ее максимальное значение достигается при $z=0$ и равно $-r_{n}^{N}(0)$, так что при $c+r_{n}^{N}(0)>0$ уравнение (34) решений иметь не может.

Докажем, что в противном случае имеется в точности одно решение уравнения (34) при $z \leqslant 0$ и при $z \geqslant 0$. Например, докажем этот факт для $z \leqslant 0$ - другой случай рассматривается вполне аналогично.

При $z \leqslant 0$ функция $g(z)-r_{n}^{N}(z)=z-r_{n}^{N}(z)$ - выпуклая, непрерывная, неубывающая, и при этом $g(z)-r_{n}^{N}(z) \rightarrow 0$, когда $z \rightarrow-\infty$ (лемма 5 ). Легко видеть, что любая функция на $(-\infty, 0]$, обладающая этими свойствами, принимает любое положительное значение, не превосходящее ее максимального значения, и притом только один раз. Поскольку, по предположению, $0<c \leqslant-r_{n}^{N}(0)=\max _{z \leqslant 0}\left\{g(z)-r_{n}^{N}(z)\right\}$, отсюда следует, что при $z \leqslant 0$ существует единственное решение уравнения $g(z)-r_{n}^{N}(z)=c$, $A_{n}^{N}$. При этом очевидно, что при $z>A_{n}^{N}$ выполняется неравенство $g(z)-r_{n}^{N}(z)>c$, т.е. $g(z)>c+r_{n}^{N}(z)$, и притом это неравенство выполняется только когда $z>A_{n}^{N}$, так как в противном случае $g(z)-r_{n}^{N}(z) \leqslant c$, в силу монотонности.

Если $c+r_{n}^{N}(0) \leqslant 0$, то $\Delta_{n}^{N}$ будет обозначать интервал $\left(A_{n}^{N}, B_{n}^{n}\right)$, а $\bar{\Delta}_{n}^{N}$ - замкнутый интервал $\left[A_{n}^{N}, B_{n}^{n}\right]$. Если же $c+r_{n}^{N}(0)>0$, то пусть, по определению, $\bar{\Delta}_{n}^{N}=\Delta_{n}^{N}=\varnothing$. Заметим, что $\Delta_{n}^{N}=\Delta_{n}^{N}(c)$ и $\bar{\Delta}_{n}^{N}=\bar{\Delta}_{n}^{N}(c)$.

Следствие 3. При выполнении условий следствия 2 его утверждение остается справедливым при замене всех ссылок на формулу (29) ссылками на формулу

$$
I_{\left\{b-z_{n} \in \Delta_{n}^{N}(c)\right\}} \leqslant 1-\psi_{n} \leqslant I_{\left\{b-z_{n} \in \bar{\Delta}_{n}^{N}(c)\right\}} .
$$

Д о к а з а т е л ь с т в о. Из леммы 6 следует, что $g\left(b-z_{n}\right)>c+$ $r_{n}^{N}\left(b-z_{n} ; c\right)$ тогда и только тогда, когда $b-z_{n} \in \Delta_{n}^{N}(c)$, а $g\left(b-z_{n}\right) \geqslant$ $c+r_{n}^{N}\left(b-z_{n} ; c\right)$ тогда и только тогда, когда $b-z_{n} \in \bar{\Delta}_{n}^{N}(c)$. Поэтому (35) эквивалентно (29).

5. Структура оптимальных последовательных критериев. Общий случай. В этом пункте мы даем характеризацию структуры оптимальных последовательных критериев, когда отсутствуют ограничения на максимальную длительность наблюдений.

Идея всего последующего - устремить максимальное допустимое число наблюдений $N$, которое в предыдущем пункте мы предполагали фиксированным, к бесконечности. При этом мы доказываем сходимость всех элементов, определяющих структуру оптимальных правил в усеченной задаче, к соответствующим элементам в неусеченной задаче (см. [21]). 
Начнем со следующей леммы.

Лемма 7. Для любого $N \geqslant 1$ и для любого $n \leqslant N$

1) $v_{n}^{N}(z) \geqslant v_{n}^{N+1}(z)$

2) $r_{n}^{N}(z) \geqslant r_{n}^{N+1}(z)$

при всех $z \in \mathbf{R}$.

Д о к а з а т е л ь с т в о. Докажем утверждение 1) по индукции по $n=N, N-1, \ldots, 1$. Пусть $n=N$. Тогда

$$
v_{N}^{N+1}(z)=\min \left\{g(z), c+\mathbf{E}_{\theta_{0}} v_{N+1}^{N+1}\left(z-q_{n}\right)\right\} \leqslant g(z)=v_{N+1}^{N+1}(z) .
$$

Предположим, что неравенство $v_{n}^{N} \geqslant v_{n}^{N+1}$ выполняется для некоторого $n, N \geqslant n>1$. Тогда

$$
\begin{aligned}
v_{n-1}^{N}(z) & =\min \left\{g(z), c+\mathbf{E}_{\theta_{0}} v_{n}^{N}\left(z-q_{n}\right)\right\} \\
& \geqslant \min \left\{g(z), c+\mathbf{E}_{\theta_{0}} v_{n}^{N+1}\left(z-q_{n}\right)\right\}=v_{n-1}^{N+1} .
\end{aligned}
$$

Таким образом, неравенство выполняется для $n-1$, что завершает индукцию.

Утверждение 2) является прямым следствием утверждения 1) в силу (27). Лемма доказана.

Так как по лемме $7 v_{n}^{N}(z)$ и $r_{n}^{N}(z)$ - невозрастающие последовательности по $N$ при каждом $z \in \mathbf{R}$, то существуют пределы (конечные или бесконечные)

$$
\begin{aligned}
& v_{n}(z)=v_{n}(z ; c)=\lim _{N \rightarrow \infty} v_{n}^{N}(z ; c), \\
& r_{n}(z)=r_{n}(z ; c)=\lim _{N \rightarrow \infty} r_{n}^{N}(z ; c) .
\end{aligned}
$$

При этом, переходя к пределу при $N \rightarrow \infty$ в (26) и $(27)$, для $n=1,2, \ldots$ получаем:

$$
\begin{aligned}
& v_{n-1}(z ; c)=\min \left\{g(z), c+\mathbf{E}_{\theta_{0}} v_{n}\left(z-q_{n} ; c\right)\right\} \\
& r_{n-1}(z ; c)=\mathbf{E}_{\theta_{0}} v_{n}\left(z-q_{n} ; c\right) .
\end{aligned}
$$

Определим $\mathscr{F}$ как класс правил остановки с конечным средним временем наблюдения при нулевой гипотезе:

$$
\mathscr{F}=\left\{\psi: \mathbf{E}_{\theta_{0}} \tau_{\psi}<\infty\right\} .
$$

Покажем, что, при выполнении условия 3 , для любого $\psi \in \mathscr{F}$ имеет место сходимость $L_{N}(\psi ; b, c) \rightarrow L(\psi ; b, c), N \rightarrow \infty$.

Лемма 8. Пусть выполнены условия 1-3 и пусть $\psi \in \mathscr{F}$. Тогда

$$
L_{N}(\psi ; b, c) \rightarrow L(\psi ; b, c)
$$

при $N \rightarrow \infty$ для любых с $>0 u b \in \mathbf{R}$. 
Д о к а з а т е л ь с т в о. Вполне аналогично доказательству леммы $4.4[21]$, с той разницей, что для доказательства сходимости

$$
\int t_{N}^{\psi} l_{N} d \mu^{N} \rightarrow 0, \quad N \rightarrow \infty
$$

вместо предположения 3 [21] мы можем использовать, в случае независимых наблюдений, более слабое условие 3 . Действительно, в обозначениях настоящей статьи

$$
\begin{aligned}
\int t_{N}^{\psi}\left|l_{N}\right| d \mu^{N} & =\mathbf{E}_{\theta_{0}} t_{N}^{\psi}\left|\min \left\{0, b-\sum_{j=1}^{N} q_{j}\right\}\right| \leqslant \mathbf{E}_{\theta_{0}} t_{N}^{\psi}\left|b-\sum_{j=1}^{N} q_{j}\right| \\
& \leqslant|b| \mathbf{P}_{\theta_{0}}\left(\tau_{\psi} \geqslant N\right)+\mathbf{E}_{\theta_{0}} t_{N}^{\psi} \sum_{j=1}^{N}\left|q_{j}\right| .
\end{aligned}
$$

Первое слагаемое в правой части (39) стремится к нулю при $N \rightarrow \infty$ в силу условия $\mathbf{E}_{\theta_{0}} \tau_{\psi}<\infty$. Для доказательства того, что второе слагаемое в правой части (39) также стремится к нулю, заметим, что, в силу условия 3 , из (22) вытекает, что ряд в левой части (22) конечен, поэтому

$$
\sum_{n=N}^{\infty} \mathbf{E}_{\theta_{0}} s_{n}^{\psi} \sum_{j=1}^{N}\left|q_{j}\right| \leqslant \sum_{n=N}^{\infty} \mathbf{E}_{\theta_{0}} s_{n}^{\psi} \sum_{j=1}^{n}\left|q_{j}\right| \rightarrow 0
$$

при $N \rightarrow \infty$. Поскольку $\mathbf{E}_{\theta_{0}} \sum_{j=1}^{N}\left|q_{j}\right|<\infty$, отсюда легко получаем, что

$$
\sum_{n=N}^{\infty} \mathbf{E}_{\theta_{0}} s_{n}^{\psi} \sum_{j=1}^{N}\left|q_{j}\right|=\mathbf{E}_{\theta_{0}} t_{N}^{\psi} \sum_{j=1}^{N}\left|q_{j}\right| \rightarrow 0
$$

при $N \rightarrow \infty$, что и требовалось. Лемма доказана.

В силу леммы 8 мы можем перейти к пределу в обеих частях неравенства (28), так что

$$
L(\psi ; b, c) \geqslant c+r_{0}(b ; c)
$$

для любого $\psi \in \mathscr{F}$, если выполнены условия 1-3. При этом, согласно лемме $4.3[21], \inf _{\psi \in \mathscr{F}} L(\psi ; b, c)=c+r_{0}(b ; c)$.

Докажем, что при выполнении условий 1-3 задача минимизации $L(\psi ; b, c)$ конечна (в терминах [21]), более точно, что справедлива следующая лемма.

Лемма 9. Если выполнень условия 1-3, то для любых $b>0, c>0$ и для любого $\psi \in \mathscr{F}$

$$
L(\psi ; b, c) \geqslant-\frac{\gamma_{1}}{8 c}
$$


Д о к а з а т е л ь с т в о. Из теоремы 1 следует, что

$$
\dot{\beta}_{\theta_{0}}(\psi, \phi) \leqslant \sqrt{\frac{\gamma_{1}}{2} \mathbf{E}_{\theta_{0}} \tau_{\psi}},
$$

поэтому

$$
L(\psi, \phi ; b, c) \geqslant c \mathbf{E}_{\theta_{0}} \tau_{\psi}-\sqrt{\frac{\gamma_{1}}{2} \mathbf{E}_{\theta_{0}} \tau_{\psi}} \geqslant-\frac{\gamma_{1}}{8 c},
$$

откуда следует (40), поскольку, согласно следствию 3.1 [21],

$$
L(\psi ; b, c)=\inf _{\phi} L(\psi, \phi ; b, c) .
$$

Лемма доказана.

3 а м е ч а н и е 3 . Из леммы 9 следует, что

$$
\inf _{\psi \in \mathscr{F}} L(\psi ; b, c)=c+r_{0}(b ; c) \geqslant-\frac{\gamma_{1}}{8 c}>-\infty
$$

для любых $b>0$ и $c>0$.

Из этого факта легко следует, что $c+r_{n}(b ; c)>-\gamma_{1} /(8 c)$ для любых $b>0, c>0$ и любого $n \geqslant 0$. Действительно, по построению, $r_{n}$ есть «функция $r_{0} \gg$ для задачи проверки гипотезы $H_{0}: \theta=\theta_{0}$ против $H_{1}: \theta>$ $\theta_{0}$ о параметре распределения процесса $X_{1}, X_{2}, \ldots$, для которого $X_{1} \sim$ $f_{\theta, n+1}, X_{2} \sim f_{\theta, n+2}, \ldots$.

Теперь теорема $4.2[21]$ принимает следующий вид.

Теорема 4. Предположим, ито выполнень условия 1-3.

Если существует $\psi \in \mathscr{F}$ такое, ито

$$
L(\psi ; b, c)=\inf _{\psi^{\prime} \in \mathscr{F}} L\left(\psi^{\prime} ; b, c\right),
$$

mo

$$
I_{\left\{g\left(b-z_{n}\right)<c+r_{n}\left(b-z_{n} ; c\right)\right\}} \leqslant \psi_{n} \leqslant I_{\left\{g\left(b-z_{n}\right) \leqslant c+r_{n}\left(b-z_{n} ; c\right)\right\}}
$$

$\mu^{n}$-почти всюду на $T_{n}^{\psi} \cap\left\{f_{\theta_{0}}^{n}>0\right\}$ для каждого $n=1,2, \ldots$

Обратно, если правило остановки $\psi$ удовлетворяет (42) $\mu^{n}$-почти всюду на $T_{n}^{\psi} \cap\left\{f_{\theta_{0}}^{n}>0\right\}$ для каждого $n=1,2, \ldots$, и при этом $\psi \in \mathscr{F}$, то оно удовлетворяет (41).

Для доказательства теоремы 4 нам потребуется следующая лемма.

Лемма 10. Функиии $r_{n}(z), n=0,1 \ldots$, определяемье соотношениями (36), обладают следуюшими свойствами:

1) $r_{n}(z) \leqslant v_{n}(z) \leqslant g(z), z \in \mathbf{R}$,

2) $r_{n}(z)$ как функиия $z \in \mathbf{R}-$ вогнута и непрерьвна,

3) $r_{n}(z)$ как функиия $z \in \mathbf{R}$ является неубывающей,

4) $z-r_{n}(z)$ как функиия $z \in \mathbf{R}$ является неубывающей,

5) $g(z)-r_{n}(z) \rightarrow 0$, когда $z \rightarrow \pm \infty$. 
Д о к а з а т е л ь с т в о. Свойства 1)-4) следуют из соответствующих свойств леммы 5 с помощью предельного перехода по $N \rightarrow \infty$ (непрерывность в свойстве 2) следует из вогнутости).

Для доказательства 5) достаточно показать, что $z-r_{n}(z) \rightarrow 0$ при $z \rightarrow-\infty$ и $r_{n}(z) \rightarrow 0$ при $z \rightarrow+\infty$.

Для доказательства того, что $r_{n}(z) \rightarrow 0$ при $z \rightarrow+\infty$, в силу (38) достаточно показать, что $v_{n}(z) \rightarrow 0$ при $z \rightarrow+\infty$.

По свойству 3 ) предел $\lim _{z \rightarrow+\infty} v_{n}(z ; c)=\lambda_{n}(c)$ (в дальнейшем, для краткости, просто $\lambda_{n}$ ) существует для всех $n=1,2, \ldots$ Из (38) следует, что $\lim _{z \rightarrow \infty} r_{n-1}(z, c)=\lambda_{n}(c), n=1,2, \ldots$ Переходя к пределу при $z \rightarrow$ $\infty$ в (37), получаем, что

$$
\lambda_{n}=\min \left\{0, c+\lambda_{n+1}\right\}
$$

для любого $n=1,2, \ldots$ Из (43) очевидно, что если для какого-то $n \geqslant 1$ выполнено неравенство $\lambda_{n}<0$, то $\lambda_{n}=c+\lambda_{n+1}<0$, следовательно, $\lambda_{n+1}=c+\lambda_{n+2}<0$, и так далее для всех остальных $n$. Это мгновенно приводит к противоречию, поскольку тогда $\lambda_{n+1}=\lambda_{n}-c, \lambda_{n+2}=\lambda_{n+1}-$ $c=\lambda_{n}-2 c, \ldots, \lambda_{n+k}=\lambda_{n}-k c, \ldots$ и, следовательно, $r_{n+k-1}(0 ; c) \leqslant \lambda_{n}-k c$ для любого $k \geqslant 1$, что противоречит тому, что $r_{n+k-1}(0 ; c) \geqslant-\gamma_{1} /(8 c)-c$ для любого $k \geqslant 1$ (см. замечание 3 ).

Следовательно, $\lambda_{n}(c)=\lim _{z \rightarrow \infty} r_{n-1}(z ; c)=0$ для любого $n \geqslant 1$.

Рассмотрим теперь случай $z \rightarrow-\infty$. Легко видеть, что

$$
v_{n-1}^{N}(z ; c)-z=\min \left\{\min \{0,-z\}, c+\mathbf{E}_{\theta_{0}}\left(v_{n}^{N}\left(z-q_{n} ; c\right)-\left(z-q_{n}\right)\right)\right\},
$$

откуда, переходя к пределу при $N \rightarrow \infty$, получаем

$$
v_{n-1}(z ; c)-z=\min \left\{\min \{0,-z\}, c+\mathbf{E}_{\theta_{0}}\left(v_{n}\left(z-q_{n} ; c\right)-\left(z-q_{n}\right)\right)\right\},
$$

где, согласно свойству 4) леммы $3, v_{n}(z ; c)-z$ - невозрастающие функции $z$ для всех $n=1,2, \ldots$. Раз так, существуют пределы $\lim _{z \rightarrow-\infty} v_{n}(z ; c)-z=\lambda_{n}(c) \leqslant 0$ (пусть, для краткости, $\lambda_{n}=\lambda_{n}(c)$ ). Точно так же, как и выше, переходя к пределу при $z \rightarrow-\infty$ в (44), получаем

$$
\lambda_{n}=\min \left\{0, c+\lambda_{n+1}\right\},
$$

$n=1,2, \ldots$. Точно так же, как и выше, предполагая, что какое-то $\lambda_{n}$ строго меньше 0 , получаем, что $\lambda_{n+k}=\lambda_{n}-k c \rightarrow-\infty$ при $k \rightarrow \infty$. Следовательно, для любого $z \leqslant 0$ выполнено неравенство $r_{n+k-1}(z ; c)-$ $z \leqslant \lambda_{n}-k c$ (согласно свойству 4) леммы 10). В частности, полагая $z=0$, получаем $r_{n+k-1}(0 ; c) \leqslant \lambda_{n}-k c$ для всех $k=1,2, \ldots$, т.е. снова противоречие с тем, что $r_{n}(0 ; c)$ ограничены снизу одной константой для Bcex $n=0,1,2, \ldots$. 
Следовательно, $\lambda_{n}=\lim _{z \rightarrow-\infty}\left(r_{n-1}(z ; c)-z\right)=0$ для любого $n=$ $1,2, \ldots$. Лемма доказана.

Д о к а з а т е ль с т в о т е о р е м ы 4. Необходимость непосредственно следует из теоремы 4.2 [21]. Для доказательства достаточности требуется показать только, что

$$
\int t_{n}^{\psi}\left(l_{n}-V_{n}\right) d \mu^{n} \rightarrow 0
$$

при $n \rightarrow \infty$ (см. (4.16) в [21]).

Из (30) следует, что $V_{n}=v_{n}\left(b-z_{n}\right) f_{\theta_{0}}^{n}$. Кроме того, мы знаем, что $l_{n}=g\left(b-z_{n}\right) f_{\theta_{0}}^{n}$. Поэтому интеграл в $(45)$ совпадает с

$$
\begin{aligned}
\int t_{n}^{\psi}\left(l_{n}-V_{n}\right) d \mu^{n} & =\mathbf{E}_{\theta_{0}} t_{n}^{\psi}\left(g\left(b-z_{n}\right)-v_{n}\left(b-z_{n}\right)\right) \\
& \leqslant \mathbf{E}_{\theta_{0}} t_{n}^{\psi}\left(g\left(b-z_{n}\right)-r_{n}\left(b-z_{n}\right)\right)
\end{aligned}
$$

(последнее неравенство выполняется в силу свойства 1) леммы 10). В силу свойств 3) и 4) той же леммы имеем для любого $z$

$$
0 \leqslant g(z)-r_{n}(z) \leqslant-r_{n}(0) \leqslant \frac{\gamma_{1}}{8 c}+c
$$

(для последней оценки мы воспользовались леммой 9 , см. замечание 3 ). Поэтому из (46) следует, что

$$
0 \leqslant \int t_{n}^{\psi}\left(l_{n}-V_{n}\right) d \mu^{n} \leqslant\left(\frac{\gamma_{1}}{8 c}+c\right) \mathbf{P}_{\theta_{0}}\left(\tau_{\psi} \geqslant n\right) \rightarrow 0
$$

при $n \rightarrow \infty$, поскольку, по условию теоремы, $\psi \in \mathscr{F}$, а значит, $\mathbf{E}_{\theta_{0}} \tau_{\psi}<$ $\infty$. Теорема доказана.

Следующая теорема следует из теоремы 4 с помощью теорем 3.1 и 3.2 из [21] и дает решение исходной условной задачи (см. введение) в классе всех последовательных критериев с правилами остановки из $\mathscr{F}$.

Теорема 5. Предположим, ито выполнены условия 1-3, и пусть $b>0$ - любое вещественное число.

Пусть $\psi$ - любое правило остановки, удовлетворяющее неравенствам

$$
I_{\left\{g\left(b-z_{n}\right)<c+r_{n}\left(b-z_{n} ; c\right)\right\}} \leqslant \psi_{n} \leqslant I_{\left\{g\left(b-z_{n}\right) \leqslant c+r_{n}\left(b-z_{n} ; c\right)\right\}}
$$

$\mu^{n}$-почти всюду на $T_{n}^{\psi} \cap\left\{f_{\theta_{0}}^{n}>0\right\}$ для каждого $n=1,2, \ldots$, и пусть решающее правило ф таково, что

$$
I_{\left\{z_{n}>b\right\}} \leqslant \phi_{n} \leqslant I_{\left\{z_{n} \geqslant b\right\}}
$$

$\mu^{n}$-почти всюду на $S_{n}^{\psi} \cap\left\{f_{\theta_{0}}^{n}>0\right\}$ для любого $n=1, \ldots, N$.

Предположим, ито $\psi \in \mathscr{F}\left(\right.$ m.е. $\left.\mathbf{E}_{\theta_{0}} \tau_{\psi}<\infty\right)$. 
Тогда критерий $(\psi, \phi)$ - локально наиболее мощный в классе всех критериев $\left(\psi^{\prime}, \phi^{\prime}\right)$ с $\psi^{\prime} \in \mathscr{F}$ в том смьлсле, ито

$$
\dot{\beta}_{\theta_{0}}(\psi, \phi) \geqslant \dot{\beta}_{\theta_{0}}\left(\psi^{\prime}, \phi^{\prime}\right)
$$

если

$$
\alpha\left(\psi^{\prime}, \phi^{\prime}\right) \leqslant \alpha(\psi, \phi) \quad u \quad \mathscr{N}_{\theta_{0}}\left(\psi^{\prime}\right) \leqslant \mathscr{N}_{\theta_{0}}(\psi) .
$$

Неравенство (49) является строгим, если строгим является хотя бы одно из неравенств (50). Если во всех неравенствах в (49) и (50) достигается равенство, то $\psi^{\prime}$ также удовлетворяет (47) $\mu^{n}$-почти всюду на $T_{n}^{\psi^{\prime}} \cap\left\{f_{\theta_{0}}^{n}>0\right\}$ для каждого $n=1,2, \ldots$ (с $\psi_{n}^{\prime}$ вместо $\left.\psi_{n}\right)$ и $\phi^{\prime}$ удовлетворяет (48) (с $\phi_{n}^{\prime}$ вместо $\left.\phi_{n}\right) \mu^{n}$-почти всюду на $S_{n}^{\psi^{\prime}} \cap\left\{f_{\theta_{0}}^{n}>0\right\}$ для любого $n=1,2, \ldots$.

Точно так же, как в предыдущем пункте, мы можем представить неравенства $g\left(b-z_{n}\right)<c+r_{n}\left(b-z_{n} ; c\right)$, определяющие форму оптимального критерия, в более простом виде. Действительно, с помощью леммы 10 нетрудно вывести, что если $c+r_{n}(z ; c) \leqslant 0$, то в каждой из областей $\{z \leqslant 0\}$ и $\{z \geqslant 0\}$ существует единственное решение уравнения

$$
c+r_{n}(z ; c)=g(z),
$$

$A_{n}=A_{n}(c) \leqslant 0$ и $B_{n}=B_{n}(c) \geqslant 0$ (см. доказательство леммы 6$)$. Обозначим в этом случае $\Delta_{n}=\Delta_{n}(c)=\left(A_{n}(c), B_{n}(c)\right)$ и $\bar{\Delta}_{n}=\bar{\Delta}_{n}(c)=$ $\left[A_{n}(c), B_{n}(c)\right]$. В случае если $c+r_{n}(z ; c)>0$, пусть $\Delta_{n}(c)=\bar{\Delta}_{n}(c)=\varnothing$. Тогда легко видеть, что (47) эквивалентно

$$
I_{\left\{b-z_{n} \in \Delta_{n}(c)\right\}} \leqslant 1-\psi_{n} \leqslant I_{\left\{b-z_{n} \in \bar{\Delta}_{n}(c)\right\}} .
$$

Поэтому из теоремы 5 вытекает следующее утверждение.

Следствие 4. При выполнении условий 1-3 утверждение теоремь 5 остается справедливым при замене всех ссылок на формулу (47) ссылками на формулу (52).

3 а м е ч а н и е 4. Если в (47) (или (52)) и, соответственно, в (48) $b<0$, то при выполнении условий теоремы 5 (с «b $<0 \gg$ вместо «b $>0 \gg)$ из теоремы $5.3[21]$ следует, что критерий $(\psi, \bar{\phi})$, где $\bar{\phi}_{n}=1-\phi_{n}, n=$ $1,2, \ldots$, является локально наиболее мощным для проверки гипотезы $H_{0}: \theta=\theta_{0}$ против альтернативы $H_{1}: \theta<\theta_{0}$ в классе всех критериев $\left(\psi^{\prime}, \phi^{\prime}\right)$ таких, что

$$
\mathbf{E}_{\theta_{0}} \tau_{\psi}^{\prime} \leqslant \mathbf{E}_{\theta_{0}} \tau_{\psi} \quad \text { и } \quad \alpha\left(\psi^{\prime}, \phi^{\prime}\right) \leqslant \alpha(\psi, \bar{\phi}) .
$$

Если $b=0$ в (47) (или (52)) и в (48), то, при выполнении всех остальных условий теоремы 5 , критерий $(\psi, \phi)$ является локально наиболее мощным для проверки гипотезы $H_{0}: \theta=\theta_{0}$ против $H_{1}: \theta>\theta_{0}$, а критерий $(\psi, \bar{\phi})$ 
является локально наиболее мощным для проверки гипотезы $H_{0}$ против $H_{1}: \theta<\theta_{0}$, в классе всех критериев, для которых

$$
\mathbf{E}_{\theta_{0}} \tau_{\psi}^{\prime} \leqslant \mathbf{E}_{\theta_{0}} \tau_{\psi}
$$

(независимо от уровня их вероятности ошибки первого рода).

3 а м е ч а н и е 5 . В данной статье мы рассматриваем только случай одномерного параметра. В [3] предпринята попытка обобщить «локальный» подход на случай многомерного параметра для критериев проверки гипотез, основанных на выборке фиксированного объема (непоследовательной). Изучение последовательных методов проверки гипотез в проблемах, подобных рассмотренным в [3], представляет собой важную, и, по-видимому, очень сложную задачу.

Некоторые частные случаи. В этом пункте мы рассмотрим задачи построения локально наиболее мощных критериев в двух частных случаях рассмотренной общей модели: в случае «периодических» наблюдений (см. [12]) и в случае «конечно-нестационарных» наблюдений (см. [16]). Случай независимых одинаково распределенных наблюдений является частным случаем обеих из этих моделей.

Рассмотрим сначала «периодический» случай, когда существует такое натуральное $T$, что $f_{\theta, n+T}=f_{\theta, n}$ для любого $n=1,2, \ldots$. В этом случае, очевидно, при выполнении условий 1 и 2 условие 3 выполняется автоматически (поскольку условие 2 гарантирует, что все $\mathbf{E}_{\theta_{0}}\left|\dot{f}_{\theta_{0}, j} / f_{\theta_{0}, j}\right|$, $j=1, \ldots, T$, конечны). Нетрудно видеть, что $v_{n}=v_{n+T}$ и $r_{n}=r_{n+T}$ для любого $n=1,2, \ldots$, поэтому решения уравнения (51) также периодичны: $A_{n}(c)=A_{n+T}(c), B_{n}(c)=B_{n+T}(c), n=1, \ldots$ Кроме того,

$$
v_{n-1}(z)=\min \left\{g(z), c+\mathbf{E}_{\theta_{0}} v_{n}\left(z-q_{n}\right)\right\}
$$

для любого $n=T, T-1, \ldots, 2$ и

$$
v_{T}(z)=\min \left\{g(z), c+\mathbf{E}_{\theta_{0}} v_{1}\left(z-q_{1}\right)\right\} .
$$

Легко видеть, что в этом случае достаточное условие оптимальности в теореме $5(\psi \in \mathscr{F})$ также выполняется, если, дополнительно к условиям 1-2, предположить, что

$$
\mathbf{P}_{\theta_{0}}\left(\sum_{j=1}^{T} q_{j}=0\right)<1 .
$$

Действительно, пусть $n=k T$ и $\xi_{i}=\sum_{j=1}^{T} q_{(i-1) T+j}, i=1,2, \ldots$ В этом случае для любого $\psi$, удовлетворяющего (52), имеет место оценка

$$
\mathbf{P}_{\theta_{0}}\left(\tau_{\psi}>n\right) \leqslant \mathbf{E}_{\theta_{0}} \prod_{j=1}^{n} I_{\left\{\sum_{i=1}^{j} q_{i} \in b-\bar{\Delta}_{j}(c)\right\}}
$$




$$
\begin{aligned}
& =\mathbf{P}_{\theta_{0}}\left(\sum_{i=1}^{j} q_{i} \in b-\bar{\Delta}_{j}(c), j=1, \ldots, n\right) \\
& \leqslant \mathbf{P}_{\theta_{0}}\left(\sum_{i=1}^{j} \xi_{i} \in b-\bar{\Delta}_{T}(c), j=1, \ldots, k\right) .
\end{aligned}
$$

При этом все $\xi_{i}, i=1,2, \ldots,-$ независимые одинаково распределенные случайные величины такие, что $\mathbf{P}_{\theta_{0}}\left(\xi_{i}=0\right)<1$, так что применима теорема Ч. Стейна [24], в силу которой, в частности, правая часть (54) имеет экспоненциальную скорость убывания, когда $k \rightarrow \infty$. Следовательно,

$$
\mathbf{E}_{\theta_{0}} \tau_{\psi}=\sum_{n=1}^{\infty} \mathbf{P}_{\theta_{0}}\left(\tau_{\psi} \geqslant n\right)<\infty
$$

т.e. $\psi \in \mathscr{F}$.

Если же условие (53) не выполняется, т.е. $\mathbf{P}_{\theta_{0}}\left(\sum_{j=1}^{T} q_{j}=0\right)=1$, то, в силу независимости $q_{j}, j=1,2, \ldots$, имеем, что $\mathbf{P}_{\theta_{0}}\left(q_{j}=0\right)=1$ для любого $j$. По построению, $v_{n}^{N}(z) \equiv g(z), r_{n}^{N}(z) \equiv g(z)$ для любого $N \geqslant 1$ и для любого $n \leqslant N$, поэтому $v_{n}(z) \equiv g(z), r_{n}(z) \equiv g(z)$ для любого $n=$ $1,2, \ldots$, так что $\mathbf{P}_{\theta_{0}}\left(\psi_{1}=1\right)=1$ для любого $\psi$, удовлетворяющего (52). Таким образом, если условие $(53)$ не выполняется, то $\mathbf{P}_{\theta_{0}}\left(\tau_{\psi}=1\right)=1$, так что тривиальным образом получаем $\psi \in \mathscr{F}$.

Итак, в периодическом случае при выполнении условий 1 и 2 любой критерий $(\psi, \phi)$ удовлетворяющий $(52)$ и $(48)$, является локально наиболее мощным в смысле теоремы 5 .

Рассмотрим теперь «конечно-нестационарный» случай. Предположим, что существует такое натуральное $k$, что $f_{\theta, j}=f_{\theta, j+1}$ для любого $j \geqslant k$ ( $k=1$ соответствует случаю независимых одинаково распределенных наблюдений). Тогда легко видеть, что $v_{n}(z ; c)=v(z ; c)$, $r_{n}(z ; c)=r(z ; c)$ (не зависят от $n$ ) для всех $n \geqslant k-1$, и при этом

$$
v(z ; c)=\min \left\{g(z), c+\mathbf{E}_{\theta_{0}} v\left(z-q_{k} ; c\right)\right\}, \quad r(z ; c)=\mathbf{E}_{\theta_{0}} v\left(z-q_{k} ; c\right),
$$

поэтому уравнение (51) для определения $A_{n}(c), B_{n}(c)$ принимает вид

$$
c+r(z ; c)=g(z) \text {, }
$$

если $n \geqslant k-1$, следовательно, $A_{n}(c)=A(c), B_{n}(c)=B(c)$ (не зависят от $n$ ), если $n \geqslant k-1$. Для остальных $n$ (если они есть) применимы рекуррентные соотношения

$$
v_{n-1}(z ; c)=\min \left\{g(z), c+\mathbf{E}_{\theta_{0}} v_{n}\left(z-q_{n} ; c\right)\right\}, \quad r_{n-1}=\mathbf{E}_{\theta_{0}} v_{n}\left(z-q_{n} ; c\right),
$$

$n=k-1, \ldots, 1$.

Разумеется, при выполнении условий 1-2, и, дополнительно, условия

$$
\mathbf{P}_{\theta_{0}}\left(q_{k}=0\right)<1
$$


тот же самый аргумент Ч. Стейна обеспечивает конечность $\mathbf{E}_{\theta_{0}} \tau_{\psi}$ для любого $\psi$, удовлетворяющего (52). Если же условие (57) не выполняется (т.е. $\left.\mathbf{P}_{\theta_{0}}\left(q_{k}=0\right)=1\right)$, то из $(55)$ следует, что $v(z ; c) \equiv g(z)$ и $r(z ; c) \equiv g(z)$, поэтому уравнение (56) иметь решения не может, так что $\Delta_{n}(c)=\bar{\Delta}_{n}(c)=\varnothing$ для любого $n \geqslant k-1$, вследствие чего правило остановки $\psi$ является усеченным $\left(\mathbf{P}_{\theta_{0}}\left(\tau_{\psi} \leqslant k-1\right)=1\right)$, т.е. $\psi \in \mathscr{F}$. Так что в конечно-нестационарном случае, при выполнении условий 1 и 2 любой критерий $(\psi, \phi)$, удовлетворяющий $(52)$ и $(48)$, является локально наиболее мощным в смысле теоремы 5 .

Из сказанного выше понятно, что случай $k=2$ представляет особый интерес, поскольку в этом случае границы области продолжения постоянны $\left(A_{n}(c)=A(c), B_{n}(c)=B(c), n=1,2, \ldots\right)$, поэтому оптимальный критерий имеет в точности ту же структуру, что и в случае независимых одинаково распределенных наблюдений (см. [8]). Подобно [8], в этом случае можно показать (в предположении (57) и дополнительно предполагая конечность информации Фишера $\left.\mathbf{E}_{\theta_{0}} q_{2}^{2}\right)$, что при любых $A<B$ критерий $(\psi, \phi)$, у которого

$$
I_{\left\{z_{n} \in(A, B)\right\}} \leqslant 1-\psi_{n} \leqslant I_{\left\{z_{n} \in[A, B]\right\}}, \quad n=1,2, \ldots,
$$

обладает свойством локальной наибольшей мощности. А именно, можно показать, что существуют константы $b, c, A<b<B, c>0$ такие, что (58) эквивалентно

$$
I_{\left\{b-z_{n} \in(A(c), B(c))\right\}} \leqslant 1-\psi_{n} \leqslant I_{\left\{b-z_{n} \in[A(c), B(c)]\right\}}, \quad n=1,2, \ldots,
$$

где $A(c), B(c)$ - решения уравнения (56).

Если константа $b$, найденная таким образом, положительна, $b>0$, то критерий $(\psi, \phi)$ с любым $\phi$, удовлетворяющим (48), является локально наиболее мощным для проверки гипотезы $H_{0}: \theta=\theta_{0}$ против альтернативы $H_{1}: \theta>\theta_{0}$; если $b<0$, то критерий $(\psi, \phi)$ с любым $\phi$, удовлетворяющим

$$
I_{\left\{z_{n}<b\right\}} \leqslant \phi_{n} \leqslant I_{\left\{z_{n} \leqslant b\right\}}, \quad n=1,2, \ldots,
$$

является локально наиболее мощным для проверки $H_{0}$ против $H_{1}: \theta<\theta_{0}$; если же $b=0$, то и тот и другой являются локально наиболее мощными, каждый для соответствующий альтернативы (см. замечание 4).

Наконец, отметим, что если распределение $q_{2}$ симметрично, то $A(c)=-B(c)$ (см. $[21$, замечание 5.3$])$, и поэтому в этом случае $b=$ $(A+B) / 2$.

Авторы благодарят рецензента за его ценные комментарии к работе, в частности, за указание на связь между условиями 1-3 и неравенством Пинскера.

Авторы выражают благодарность Александру Галкину за помощь в информационном обеспечении. 
Ан. Новиков благодарит Национальную систему исследователей (SNI CONACyT) (Мексика) за поддержку данной работы, а также Национальный совет по науке и технологии (CONACyT) (Мексика) за поддержку в рамках гранта CB-2005-C01-49854-F.

\section{СПИСОК ЛИТЕРАТУРЫ}

1. Володин И.Н. Гарантийные процедуры статистического вывода (определение объема выборки). - Исследования по прикладной математике. Казань, 1984, в. 10, c. $13-53$.

2. ДеГроот М. Оптимальнье статистические решения. М.: Мир, 1974, 492 с.

3. Новиков П. А. Локально максиминный по направлениям критерий для многомерного параметра с частично упорядоченными альтернативами. - Изв. вузов, 2011, № 1 , с. $39-48$.

4. Новиков П. А. Локально наиболее мощные последовательные критерии для марковских процессов с дискретным временем. - Теория вероятн. и ее примен., 2010, т. 55 , в. 2 , с. $369-372$.

5. Пинскер М. С. Информация и информационная устойчивость случайных величин и процессов. М.: Изд-во АН СССР, 1960, 201 с.

6. Рокафеллар Р. Выпукльй анализ. М.: Мир. 1973, 470 с.

7. Abraham J.K. The local power of sequential tests subject to an expected sample size restriction. Technical report № 38. Stanford: Stanford University, 1969.

8. Berk R. H. Locally most powerful sequential tests. - Ann. Statist., 1975, v. 3, p. 373381.

9. Ghosh M., Mukhopadhyay N., Sen P.K. Sequential Estimation. New York: Wiley, 1997, $480 \mathrm{p}$.

10. Ferguson T.S. Mathematical Statistics: A Decision Theoretic Approach. New York: Academic Press, 1967, 396 p.

11. Irle A. Sequentialanalyse. Optimale sequentielle Tests. Stuttgart: Teubner, 1990, $176 \mathrm{p}$.

12. Liu Y., Blostein S.D. Optimality of the sequential probability ratio test for nonstationary observations. - IEEE Trans. Inform. Theory, 1992, v. 38, p. 177-182.

13. Müller-Funk U. Mathematical Programming and Optimal Stopping in Sequential Testing Theory. Habilitationsschrift, Universität Freiburg, 1986.

14. Müller-Funk U., Pukelsheim F., Witting H. Locally most powerful tests for two-sided hypotheses. - Probability and Statistical Decision Theory, v. A (Bad Tatzmannsdorf, 1983). Dordrecht: Reidel, 1985, p. 31-56.

15. Novikov A. Locally most powerful two-stage tests. - Prague Stochastics 2006. Proceedings of the joint session of 7th Prague Symposium on Asymptotic Statistics and 15th Prague Conference on Information Theory, Statistic Decision Functions and Random Processes (Prague 2006). Prague: Matfyzpress, Charles University in Prague, 2006, p. 554-567.

16. Novikov A. Optimal sequential tests for two simple hypotheses based on independent observations. - Int. J. Pure Appl. Math., 2008, v. 45, № 2, p. 291-314.

17. Novikov A. Optimal sequential procedures with Bayes decision rules. - Kybernetika, 2010 , v. 46, № 4 , p. 754-770.

18. Novikov A. Optimal sequential procedures with Bayes decision rules. - Int. Math. Forum, 2010, v. 5, № 43, p. 2137-2147.

19. Novikov A. Optimal sequential multiple hypothesis tests. - Kybernetika, 2009, v. 45, № 2 , p. 309-330.

20. Novikov A. Optimal sequential tests for two simple hypotheses - Sequential Anal., 2009 , v. 28 , № 2 , p. 188-217. 
21. Novikov A., Novikov P. Locally most powerful sequential tests of a simple hypothesis vs. one-sided alternatives. - J. Statist. Plann. Inference, 2010, v. 140, № 3, p. 750765.

22. Roters $M$. Locally most powerful sequential tests for processes of the exponential class with stationary and independent increments. - Metrika, 1992, v. 39, № 3-4, p. $177-183$.

23. Schmitz N. Optimal Sequentially Planned Decision Procedures. New York: SpringerVerlag, 1993, 207 p. (Lecture Notes in Statist., v. 79.)

24. Stein C. A note on cumulative sums. - Ann. Math. Statist., 1946, v. 17, p. 498-499.

25. Wald A. Statistical Decision Functions. New York: Wiley, 1950, 179 p.

Поступила в редакцию 3.VIII.2009

Исправленный вариант 28.IV.2011 